\title{
Age-Related Changes to Vestibular Heave and Pitch Perception and Associations with Postural Control
}

\section{Grace A Gabriel}

Department of Psychology, University of Toronto, Toronto, ON

\section{Laurence R Harris}

Department of Psychology and Centre for Vision Research, York University, Toronto, ON

\section{Joshua J Gnanasegaram}

KITE - Toronto Rehabilitation Institute, University Health Network, Toronto, ON

\section{Sharon L Cushing}

Department of Otolaryngology- Head \& Neck Surgery Hospital for Sick Children, Toronto, ON

\section{Karen A Gordon}

Department of Otolaryngology- Head \& Neck Surgery Hospital for Sick Children, Toronto, ON

\section{Bruce C Haycock}

KITE - Toronto Rehabilitation Institute, University Health Network, Toronto, ON Jennifer L Campos ( $\sim$ jennifer.campos@uhn.ca )

KITE - Toronto Rehabilitation Institute, University Health Network, Toronto, ON

\section{Research Article}

Keywords: self-motion, aging, thresholds, rotation, translation

Posted Date: October 27th, 2021

DOI: https://doi.org/10.21203/rs.3.rs-969657/v1

License: (c) (i) This work is licensed under a Creative Commons Attribution 4.0 International License. Read Full License

Version of Record: A version of this preprint was published at Scientific Reports on April 19th, 2022. See the published version at https://doi.org/10.1038/s41598-022-09807-4. 


\section{Age-Related Changes to Vestibular Heave and Pitch Perception and Associations with Postural Control}

Grace A. Gabriel ${ }^{1,2}$, Laurence R. Harris ${ }^{3}$, Joshua J. Gnanasegaram ${ }^{1}$, Sharon L. Cushing ${ }^{4-6}$, Karen A. Gordon ${ }^{4-6}$, Bruce C. Haycock ${ }^{1,7}$, Jennifer L. Campos ${ }^{1,2, *}$

${ }^{1}$ KITE - Toronto Rehabilitation Institute, University Health Network, Toronto, ON, Canada

${ }^{2}$ Department of Psychology, University of Toronto, Toronto, ON, Canada

${ }^{3}$ Department of Psychology and Centre for Vision Research, York University, Toronto, ON, Canada

${ }^{4}$ Department of Otolaryngology- Head \& Neck Surgery Hospital for Sick Children, Toronto, ON, Canada

${ }^{5}$ Department of Otolaryngology- Head \& Neck Surgery, University of Toronto, Toronto, ON, Canada

${ }^{6}$ Archie's Cochlear Implant Laboratory, Hospital for Sick Children, Toronto, ON, Canada

${ }^{7}$ University of Toronto Institute for Aerospace Studies, Toronto, ON, Canada

Word Count Main Text: 7333 words

Word Count Abstract: 196 words

24 This work has been presented at the International Multisensory Research Forum (June 2018), the

25 Toronto Rehabilitation Institute Research Day (January 2018) and the Lake Ontario Visionary

26 Establishment (February 2019). Some of the data in this manuscript also appears in Gabriel et al.

27 [1]. *Correspondence: Jennifer L. Campos, 500 University Avenue, Toronto ON, Canada. M5G

28 2A2.jennifer.campos@uhn.ca 
Acknowledgements

2 We would like to acknowledge Dr. Paul Mick, Dr. Peter Grant, and Dr. M. Kathleen Pichora-

3 Fuller for their helpful earlier discussions. We are also grateful for Rebecca Benjamin and

4 Melissa Hazen for their assistance in the collection and interpretation of VEMP and vHIT data.

5 We would also like to thank Susan Gorski and Rob Shewaga for technical assistance in operating

6 the motion platform. 


\section{Abstract}

Falls are a common cause of injury in older adults (OAs), and age-related declines across

3 the sensory systems are associated with an increased risk of falls. The vestibular system is

4 particularly important for maintaining balance and supporting safe mobility, and aging has been

5 associated with declines in vestibular end-organ functioning. However, few studies have

6 examined potential age-related differences in vestibular perceptual sensitivities or their

7 association with postural stability. Here we used an adaptive-staircase procedure to measure

8 detection and discrimination thresholds in 19 healthy OAs and 18 healthy younger adults (YAs),

9 by presenting participants with passive heave and pitch movements on a motion-platform in the

10 dark. We also examined participants' postural stability under various standing-balance

11 conditions. Associations among these postural measures and vestibular perceptual thresholds

12 were further examined. Ultimately, OAs showed larger heave and pitch detection thresholds

13 compared to YAs, and larger perceptual thresholds were associated with greater postural sway,

14 but only in OAs. Overall, these results suggest that vestibular perceptual sensitivity declines with

15 older age and that such declines are associated with poorer postural stability. Future studies could

16 consider the potential applicability of these results in the development of screening tools for falls

17 prevention in OAs.

18 Keywords: self-motion, aging, thresholds, rotation, translation 


\section{Introduction}

Falls are the most common cause of fatal and non-fatal injuries in adults over the age of 65 years [2], [3] and age-related declines across sensory systems (e.g., vision, hearing, proprioception, vestibular) contribute to an increased risk of falls. The vestibular system is particularly important for maintaining balance and supporting safe mobility [4], [5]. It is wellestablished that vestibular functioning changes with age as evidenced by physiological changes and changes to peripheral vestibular end-organ measures [6]-[8]. There are also well-known agerelated behavioral changes, such as declines in postural control, that occur with older age [9][15]. However, much less is known about age-related changes to vestibular perception [16]-[20] (but see [21]for a review) [16]-[20]and how vestibular perceptual abilities are associated with postural stability in older adults. In this study, we examine age-related differences in vestibular detection and discrimination thresholds during passive heave and pitch movements in the dark and explore how these percepts are associated with posturography measures (i.e., center of pressure path length, velocity, root-mean-squared velocity).

\section{Age-Related Changes in Vestibular End-Organ Structure and Functioning}

The vestibular system comprises five distinct organs: three semicircular canals that detect rotational motion in all three axes (yaw, pitch, and roll), as well as two otolith organs that detect linear acceleration and gravity in the vertical or heave axis (saccule), and in the horizontal, or surge and sway axes (utricular, and some saccular) [22]. These vestibular end-organs send sensory information to the brain through the vestibulocochlear nerve, which informs the perception of self-motion [23] for a review).

Age-related changes to the vestibular system have been evaluated by examining the 
1 vestibular end-organs histologically and microscopically [24]-[26] (but see [6]-[8], [27] for

2 reviews). These studies have demonstrated that aging is associated with declines in end-organ

3 integrity, including deterioration of the otoconia in the otolith organs, particularly in the utricle

4 [28] and a loss of Type I [29] and Type II hair cells [24], [29]-[32], with the semicircular canals

5 being particularly susceptible to Type I hair cell loss [32], [33]. There is also evidence of

6 degeneration of the vestibular ganglion with aging [34], [35], especially the superior vestibular

7 nerve [36] which relays afferent superior (i.e., pitch) and lateral (i.e., yaw) semicircular canal

8 information to the brain.

9 Within clinical settings, saccular, utricular, and semicircular canal functioning are often

10 assessed using cervical vestibular-evoked myogenic potentials (cVEMP), ocular VEMPs

11 (oVEMP) and video head impulse testing (vHIT), respectively. Reported age-related changes to

12 these measures include decreased cVEMP amplitudes [37], [38] and increased oVEMP latencies

13 [39], which suggest decline in vestibular functioning. There is also evidence of slightly lower

14 vHIT gains in older adults over 85 years of age compared to younger adults [37], [40], [41][37],

15 [40], [41], although many studies also show that vHIT is resistant to age-related effects [42],

16 [43]. Less, however, is understood about how age-related changes to end-organ functioning, as

17 measured by VEMP and vHIT, are associated with age-related changes in self-motion perception

18 or vestibularly-informed behaviors such as balance and postural control [44]-[46].

\section{Age-Related Changes in Postural Control and Vestibular Perception}

A wealth of previous research has characterized age-related changes to postural control

22 [47]-[51]. For instance, older adults demonstrate poorer standing balance (e.g., longer center of

23 pressure, or COP, path lengths and greater sway velocity) during static posturography tasks 
1 compared to younger adults, particularly with eyes-closed [52], while standing on compliant or

2 unstable surfaces [12], [53], or during balance perturbations (e.g., [54]).

3 During passive movements in the dark, self-motion perception is thought to be largely

4 informed through vestibular inputs. Under such conditions younger adults have demonstrated an

5 ability to detect, estimate, and discriminate rotational velocity [18], [55], linear heading direction

6 [20], [56]-[60], distance travelled [59], [61]-[63], and target-relative spatial updating [64]-[69].

7 There is emerging, but limited evidence suggesting that vestibular perception changes with older

8 age. For instance several studies have shown that, compared to younger adults, older adults

9 demonstrate higher direction discrimination thresholds for sway, heave, and roll-tilt [16], [17],

10 [19], [21], [70], [71] but not yaw [16], [70]. These studies have also shown that younger and

11 older adults do not demonstrate differences in their ability to detect movements in yaw, or in

12 their ability discriminate between two passively-applied yaw rotations [18]. Greater age-related

13 differences in direction-discrimination thresholds have been reported for linear movements

14 compared to rotational movements [16]. No studies to our knowledge, however, have examined

15 age-related perceptual changes in the pitch direction, although previous studies have suggested

16 that older adults may have a biased perception of verticality (with "backward disequilibrium

17 syndrome") and exhibit a backwards-tilted bias in their subjective postural vertical, when

18 compared to healthy control participants [72]-[74]. As such, examining pitch perception in older

19 adults may be particularly informative given that forward-tilt detection has been described as a

20 potential predictor of falls risk in older adults [75], [76]. Likewise, falls and recovery from falls,

21 especially in older adults, may be associated with motion in the z-plane, as elevating and

22 lowering strategies are the most common strategies used to recover from a fall due to tripping 
1 [75]-[78]. Therefore, we also investigated the extent to which there may be age-related

2 differences in the ability to detect, and discriminate between, vertical linear (i.e., heave) motions.

3 Furthermore, it might be expected that less sensitive vestibular perception may have

4 negative implications for behaviors that are informed by self-motion perception, such as standing

5 balance. Yet, few studies have examined the extent to which age-related changes in balance and

6 postural control are associated with measures of vestibular self-motion perception. It has recently

7 been shown that in younger and middle-aged adults, different measures of COP path length are

8 related to vestibular perceptual thresholds for many translational and rotational movements [79].

9 The results showed that vestibular perceptual thresholds in the lateral plane were positively

10 associated with COP path length. In older adults specifically, some research has indicated that

11 higher roll-tilt thresholds are associated with a greater risk of failing the most difficult condition

12 of a Romberg Balance Test (i.e., quiet stance on a compliant surface with eyes closed; [16], [17],

13 [19]). While failure to successfully complete this type of balance test is an important indicator of

14 poor postural control, the binary nature of "pass" and "fail" tasks could conceal subtler

15 differences or declines in postural stability which may, nonetheless, be important predictors of

16 mobility or falls risk [80]-[83]. Higher resolution spatial and temporal measures of sway could

17 allow for the examination of finer differences in postural control which may not be severe

18 enough to cause full balance failures. It would also provide the opportunity to relate such

19 differences to vestibular perceptual thresholds in older and younger adults.

20 To our knowledge, associations between vestibular perceptual thresholds (e.g., direction

21 discrimination, detection, magnitude-discrimination) and more precise spatial or temporal

22 features of postural sway (e.g., COP path length and velocity) have not been examined in healthy

23 older adults. In general, better characterizing vestibular perceptual sensitivities of older adults 
1 across a range of motion types and axes, as well as understanding how these perceptual abilities

2 are associated with high resolution measures of posture could help to clarify the extent to which

3 age-related declines in vestibular function (e.g., presbyvestibulopathy; [84]) contribute to

4 balance problems and falls risk.

6 Current Study

7 In this study, we measured peripheral vestibular end-organ functioning using vHIT and

8 VEMPs in older adults, as well as behavioral balance functioning (posturography during quiet

9 standing) and vestibular perception (two-interval detection and magnitude discrimination

10 thresholds) in younger and older adults. We also examined associations between perceptual

11 thresholds and posturography measures in each age group. Specifically, by passively moving

12 participants using a 6 degrees-of-freedom motion platform we measured movement detection

13 and magnitude discrimination thresholds during heave translation, which stimulates the saccule,

14 and during pitch rotation, which stimulates both anterior and posterior canals, as well as the

15 saccule and utricle. We also used a static posturography task to assess postural stability and

16 performed a series of exploratory correlations between vestibular perceptual thresholds and

17 posturography measures for each age group.

\section{Methods}

\section{Participants}

19 healthy older adults $\left(M_{\mathrm{age}}=70.47\right.$ years, $S D=5.64$, range $=65-89$ years, 11 females,

228 males $)$ and 18 younger adults $\left(M_{\text {age }}=26.00\right.$ years, $S D=4.27$, range $=20-34$ years, 13

23 females, 5 males) completed the study. All participants gave written informed consent. A subset 
1 of the older adult participants were included as a control sample in Gabriel et al. [1]. Participants

2 were recruited from the community using posters, social media posts, websites, and through an

3 existing participant database. These individuals were eligible to participate if they did not have a

4 history of stroke, seizure, disabling musculoskeletal disorder, acute psychiatric disorder,

5 dementia, mild cognitive impairment, clinically diagnosed vestibular disorders (e.g., Meniere's

6 disease), hearing loss, or if they were unable to provide informed consent. All older adults

7 obtained above cut-off scores on the Montreal Cognitive Assessment for mild cognitive

8 impairment (MoCA; i.e., $\geq 26$ points; [85][85]). All methods in this study were conducted in

9 accordance with the Declaration of Helsinki and approved by the University Health Network's

10 Research Ethics Board (Protocol \#: 18-6123.0), the University of Toronto Research Ethics Board

11 (Protocol \#: 00037394), and The Hospital for Sick Children Research Ethics Board (Protocol \#:

12 1000056920).

\section{Baseline Assessment Session Tests}

14 Older adult participants first underwent a series of baseline sensory (i.e., hearing and

15 vestibular), cognitive, and balance assessments (Table 1), each described in detail in the

16 following sections.

17 Table 1.

18 Summary of baseline assessments measured in the older adult participants

19

\begin{tabular}{ll}
\hline Baseline Measure & M (SD) \\
\hline $\begin{array}{c}\text { Hearing PTA Threshold } \\
\text { Cognition }\end{array}$ & $11.29(5.63)$ \\
MoCA $^{2}(/ 30$ total) & \\
\hline Vestibular End-Organ & $27.39(1.46)$ \\
vHIT $^{3}$ (total $\left.n\right)$ & 10 \\
vHIT (right ear) & $0.96(0.19)$ \\
vHIT (left ear) $_{\text {cVEMP }^{4}(\text { total } n)}$ & $0.89(0.15)$ \\
\end{tabular}




\begin{tabular}{cl} 
cVEMP (present, right ear, $n)$ & $87 \%$ \\
$\operatorname{cVEMP}($ present, left ear, $n)$ & $67 \%$ \\
$\operatorname{oVEMP}^{5}(n)$ & 14 \\
$\operatorname{oVEMP}$ (present, right ear, $n)$ & $27 \%$ \\
oVEMP (present, left ear, $n)$ & $27 \%$ \\
\hline
\end{tabular}

\section{Balance}

$\begin{array}{ll}\mathrm{ABC}^{6}(/ 100 \%) & 94.82(5.15) \\ \text { Fell in the last year }(n) & 3 \\ \text { Near fall(s) in last year }(n) & 2 \\ \text { Fear of falling }(n) & 2\end{array}$

${ }^{1}$ PTA $=$ Pure Tone Average; frequencies tested: 500, 1000, 2000, and $4000 \mathrm{~Hz}$, inclusive, with a cut-off threshold above $25 \mathrm{~dB}$ HL.

${ }^{2} \mathrm{MoCA}=$ Montreal Cognitive Assessment ( $\max$ score $=30$; clinical cut off $\leq 26 \mathrm{pts}$ )

${ }^{3} \mathrm{vHIT}=$ Video Head Impulse Test. 9 participants were not able to come back to complete this session. Median gain for $60 \mathrm{~ms}$ reported. One participant obtained a median gain below the 0.7 cut off score at $60 \mathrm{~ms}$ (they obtained 0.67 , in the left ear).

${ }^{4} \mathrm{cVEMP}=5$ participants were not able to come back to complete this session. Cervical Vestibular Evoked Myogenic Potential

${ }^{5}$ oVEMP $=5$ participants were not able to come back to complete this session. Ocular Vestibular Evoked Myogenic Potential

${ }^{6} \mathrm{ABC}=$ Activities-specific Balance Confidence Scale $(\max$ score $=100 \%)$

\section{Hearing}

Given that declines in vestibular functioning may be associated with age-related hearing loss [1], [86]-[91] older adult participants were screened for hearing abilities. Audiometric testing was completed as per guidelines established by the International Organization of Standardization (ISO; ISO 8253-1, 1989). Pure-tone audiometry was used to determine audiometric hearing thresholds using a Grason-Stadler 61 Clinical Audiometer (GSI-61; GrasonStadler Inc., Eden Prairie, MN) and Telephonics TDH-50P headphones (Telephonics Corporation, Farmindale, NY). Testing was performed in a double-walled sound-attenuating booth (Industrial Acoustics Company, Inc., New York, NY). Frequencies tested were between $250 \mathrm{~Hz}$ and $8000 \mathrm{~Hz}$, inclusive. Binaural pure-tone audiometric (PTA) thresholds below $25 \mathrm{~dB}$

HL, when averaged across the $500 \mathrm{~Hz}, 1000 \mathrm{~Hz}, 2000 \mathrm{~Hz}$, and $4000 \mathrm{~Hz}$ frequencies, were 4 considered normal. Three older adult participants could not come into the lab to have their 
1 hearing tested, but these older adults had self-reported normal hearing and their vestibular

2 threshold and posturography data did not differ significantly from the rest of the older adults.

\section{Cognition.}

4 Mild cognitive impairment was screened for using the MoCA. The MoCA is a rapid test

5 designed to screen individuals for mild cognitive impairment. The test assesses general cognitive

6 abilities, by examining several domains of cognitive functioning including attention, executive

7 function, memory, and language and is scored out of a total of 30 points. In this study, level-of-

8 education adjusted scores are reported and all participants obtained a score of 26 or higher

9 (common cut-off for mild cognitive impairment).

$10 \quad$ Vestibular.

11 Vestibular end-organ functioning was assessed using vHIT and VEMPs to measure

12 semicircular canal and otolith organ functioning respectively. vHIT assesses semicircular canal

13 (in this case lateral canals) functioning by measuring (and here, reporting) participants'

14 vestibulo-ocular reflex (VOR). A gain less than 0.7 suggests impaired semicircular canal

15 functioning [42], [93]-[95].

16 VEMPs are a measure of otolith organ functioning that exploit the sound-sensitive fibers

17 contained in the saccule and utricle [96]. cVEMPs were scored as "present", indicating normal

18 otolith function, if activity presented a P1 peak at 10-25 ms, followed by an N1 trough at 20-40

19 ms. Absence of this peak was coded as "absent" and indicated potential dysfunction of the otolith

20 organs. oVEMPs were coded as present if the test demonstrated an N1 at 8-20 ms, followed by a

21 P1 between 15-30 ms. These latency ranges were based on the possibility that peak latencies may

22 change with older age [8], [97], [98]. 
Details regarding vHIT and VEMP testing procedures can be found in the online

2 supplemental materials (S.1).

\section{Balance.}

4 Self-reported balance function during day-to-day tasks was measured using the

5 Activities-specific Balance Confidence (ABC) scale [99]. Excellent perceived balance was

6 scored by participants on each item as $100 \%$, and very poor subjective balance as $0 \%$.

7 Posturography tasks were also used to assess standing balance during the experimental session

8 (see details below).

9 Demographics and Health History Questionnaire.

A questionnaire recording the participants' demographics and medical background was

11 administered. Items included questions regarding education, dizziness, history or presence of

12 vestibular disorders, fear of falls, history of falls, smoking and drinking habits, subjective

13 cognitive decline, heart disease and other vascular or neurological health problems.

\section{Experimental Session}

\section{Vestibular psychophysics task}

Stimuli and Apparatus. The vestibular psychophysics tasks were performed within the

17 KITE - Toronto Rehabilitation Institute's Challenging Environment Assessment Laboratory

18 (CEAL). CEAL contains a $6.0 \mathrm{~m} \times 5.6 \mathrm{~m} \times 4.1 \mathrm{~m}$ enclosed laboratory mounted on a 6-degrees-

19 of-freedom hexapod motion base (i.e., capable of moving in all linear directions and rotating

20 around pitch, yaw, and roll axes), with 60 " actuator arms allowing tilting up to $100 \mathrm{deg} / \mathrm{s}^{2}$ in the

21 pitch axis, and $8 \mathrm{~m} / \mathrm{s}^{2}$ in the heave direction (see Figure 1).

22 For this study, the laboratory was outfitted with a specially constructed chair designed to

23 minimize participants' head and body movements. The chair was cushioned with foam to reduce 
1 vibrotactile feedback. Participants were secured in their seats by means of a four-point harness

2 and had their feet resting on foam mats at the base of the chair to restrict leg-movement and to

3 also reduce vibrotactile cues to the feet during the task. A neck pillow was used to further limit

4 proprioceptive feedback through incidental movement of the head or neck. Finally, participants

5 were blindfolded and wore noise-cancelling headphones that presented white-noise throughout

6 each block to limit the sound created by the hydraulics of the motion base. Lights were also

7 dimmed inside the lab for the duration of the experiment.

The experimenter sat inside the lab with the participants but communicated with them

9 through a microphone feeding into the headphones. The goal of this setup was to reduce as many

10 sensory cues to motion as possible to isolate information about the passive movements to those

11 arising primarily from the vestibular system.

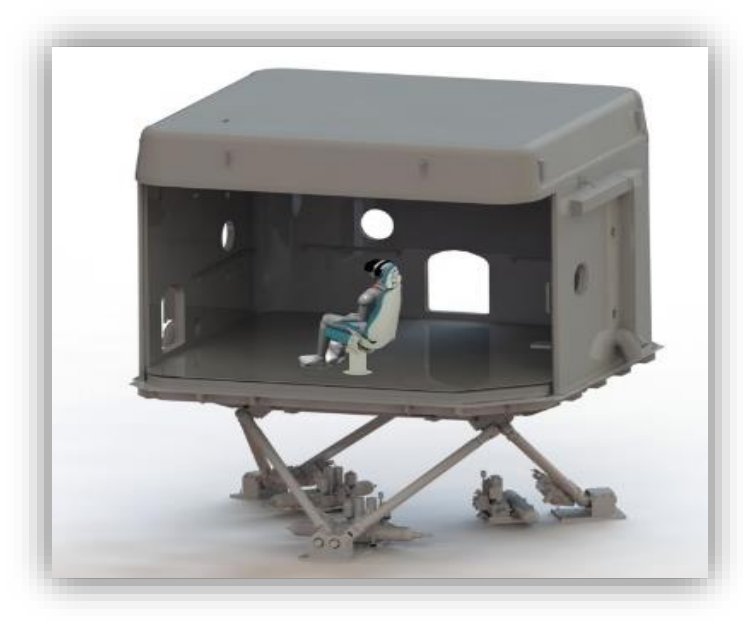

Figure 1. Vestibular Psychophysics setup. Schematic of the laboratory setup for the psychophysical task including the 6-degrees-of-freedom motion platform.

Movement Specifications. There were four conditions in the main psychophysical task:

14 heave detection, heave discrimination, pitch detection, and pitch discrimination. The point of

15 rotation for pitch movements was at the approximate center of the head. Each trial consisted of

16 1) a standard movement and 2) a comparison movement. For detection, the platform remained 
1 stationary during the "standard movement" (see Table 2 for movement specifications).

2 Magnitudes are stated as peak accelerations for both heave $\left(\mathrm{m} / \mathrm{s}^{2}\right)$ and pitch $\left(\mathrm{deg} / \mathrm{s}^{2}\right) \mathrm{motions}$.

$4 \quad$ Table 2. Initial peak accelerations used for the psychophysical tasks.

\begin{tabular}{|c|c|c|}
\hline Movement Type & Detection Task & Discrimination Task \\
\hline \multirow[t]{2}{*}{ Heave } & 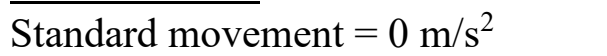 & $\overline{\text { Standard movement }}=1.0 \mathrm{~m} / \mathrm{s}^{2}$ \\
\hline & $\begin{array}{l}\text { Initial comparison movement }=0.5 \\
\mathrm{~m} / \mathrm{s}^{2}\end{array}$ & $\begin{array}{l}\text { Initial comparison movement }=1.5 \\
\mathrm{~m} / \mathrm{s}^{2}\end{array}$ \\
\hline \multirow[t]{2}{*}{ Pitch } & Standard movement $=0 \mathrm{deg} / \mathrm{s}^{2}$ & Standard movement $=20 \mathrm{deg} / \mathrm{s}^{2}$ \\
\hline & $\begin{array}{l}\text { Initial comparison movement }=3 \\
\mathrm{deg} / \mathrm{s}^{2}\end{array}$ & $\begin{array}{l}\text { Initial comparison movement }=26 \\
\mathrm{deg} / \mathrm{s}^{2}\end{array}$ \\
\hline
\end{tabular}

5 Note. The initial comparison movements in this table represent the initial acceleration values.

6 These values changed throughout the session as a function of the PEST procedure outlined in the 7 text.

Each full trial consisted of a standard movement and a comparison movement presented

10 in a random order [100]. The movements all followed the same profile. The platform was

11 oscillated at $0.5 \mathrm{~Hz}$ either in pitch or heave, beginning at rest in a central position with the

12 participant sitting upright. The platform was then oscillated sinusoidally around this position

13 with a peak velocity that increased along a raised cosine velocity envelope reaching the desired

14 value after three seconds. The platform then oscillated with this peak velocity, and the

15 corresponding peak accelerations, for five seconds (Figure 2, yellow shaded area). The peak

16 velocity then changed in magnitude along a raised cosine velocity envelope for three seconds

17 (Figure 2, grey shaded area) and then oscillated with a second peak velocity and acceleration for

18 another five seconds (i.e., the second movement of the trial; Figure 2, blue shaded), before

19 returning back to rest for three seconds. The motion base then rested for one second, or until the

20 participant made their response. Each complete trial lasted approximately 20 seconds (see Figure

212 for an example of a full heave discrimination trial). 


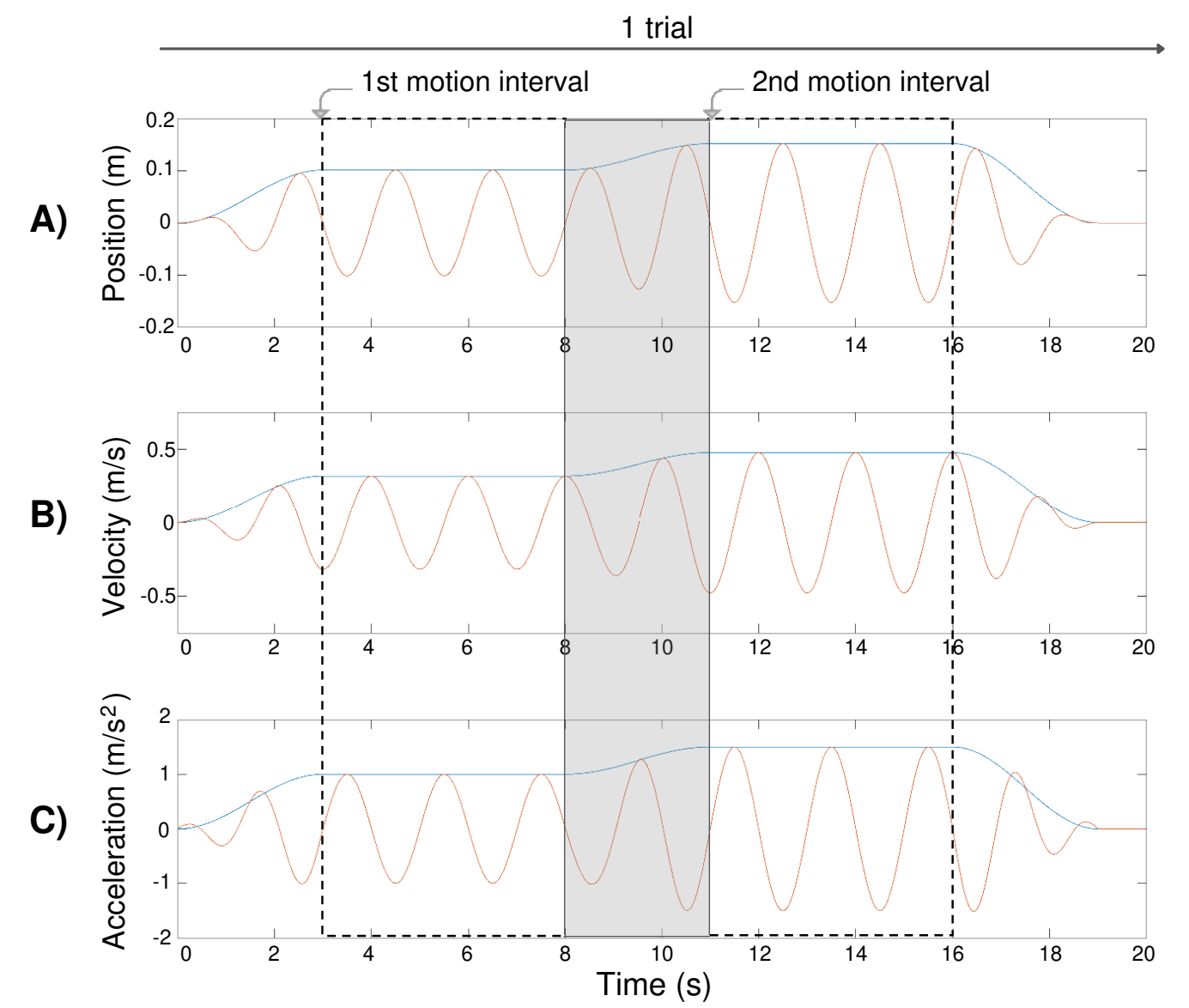

Figure 2. Diagrammatic representation of a single heave discrimination trial. A) the position $(\mathrm{m})$ relative to the upright start position, B) the velocity $(\mathrm{m} / \mathrm{s})$ and $\mathrm{C})$ the acceleration $\left(\mathrm{m} / \mathrm{s}^{2}\right)$ of the motion base. For pitch trials, displacement was measured in degrees, velocity in $\mathrm{deg} / \mathrm{s}$, and acceleration in $\mathrm{deg} / \mathrm{s}^{2}$. The yellow shaded area highlights the first movement $(5 \mathrm{~s}$; here, standard movement), the grey shaded area represents the fade-in between the first and second movement ( $3 \mathrm{~s})$, and the blue region represents the second movement $(5 \mathrm{~s}$; here, comparison movement). The unshaded white regions represent the fade from no motion to the first movement ( $3 \mathrm{~s})$, or from the second movement to no motion (3s), and rest (1s). magnitude discrimination task for each of the two motion types - pitch and heave - resulting in four psychophysical conditions in total. The four conditions (heave detection, heave discrimination, pitch detection, pitch discrimination) were presented in a random order across 
1 participants to protect against effects of carryover, practice, or fatigue across conditions. Breaks

2 were provided on an as-needed basis. In total, these four conditions took approximately one hour

3 per participant to complete.

4 Detection. Each individual trial in the detection condition was comprised of two

5 intervals: 1) a motion interval (in pitch or heave, depending on the condition) and 2) a no motion

6 interval. The order of these two motion intervals were randomized across trials within a

7 condition. After both intervals were presented (demarcated by a spoken "one" or "two", via the

8 headphones, at the point of the peak acceleration), participants were asked to state out loud

9 which of the two intervals was the one in which they had moved ("one" or "two"). The

10 acceleration of the motion presented was varied using a Parametric Estimation by Sequential

11 Testing procedure (PEST, [101]) until the participant's detection threshold was reached. PEST is

12 an adaptive staircase procedure that uses a set of rules to converge on perceptual thresholds

13 corresponding quickly and efficiently to where participants were $70.7 \%$ correct, in this instance

14 [102]. To vary the values presented logarithmically, the base-10 logarithms of the acceleration

15 values (beginning with the initial comparison movement value; see Table 2) were used by the

16 PEST and the PEST's log output was exponentiated into peak acceleration values before being

17 fed to the platform's motors.

18 Using the logged acceleration values, the PEST decreased the magnitude of the

19 subsequent trial by a single "step" (defined below) if the participant responded correctly on two

20 consecutive trials. If they responded incorrectly once, the value would increase by one step. The

21 initial step sizes were $\log (0.1)$ for the heave condition and $\log (0.2)$ for the pitch condition. On

22 the third step in the same direction, the size of the step doubled. This was unless the third step in

23 the same direction was before the last reversal, in which case the rule was to wait until the fourth 
1 step. A change in direction (e.g., going from an increase to a decrease in peak acceleration, or

2 step) represented a "reversal". Eight reversals or 60 trials, whichever came first, signaled the end

3 of a block of trials (i.e., vestibular threshold reached). The $70.7 \%$ correct threshold was

4 calculated by averaging the accelerations of the last three reversals [102].

Discrimination. As in the detection condition, the discrimination condition used a similar

6 PEST procedure to determine participants' movement discrimination thresholds, except with the

7 PEST being applied to the delta relative to the standard movement rather than the comparison

8 amplitude. Participants were required to discriminate (i.e., report which motion felt larger)

9 between two sequentially presented movements of different magnitudes: 1) a standard movement

10 in pitch or heave and 2) a comparison movement, also in pitch or heave. These two movement

11 intervals were separated by a sound file comprised of a spoken "one" or "two" presented through

12 their headphones. Peak accelerations of the comparison movement interval were determined via

13 the above-described PEST procedure using the same initial step sizes and the same termination

14 criteria. The $70.7 \%$ correct discrimination thresholds were calculated by averaging the

15 accelerations of the last three reversals [102].

$16 \quad$ Posturography Task

17 Participants also completed a posturography task to assess their standing balance. In this

18 task, participants stood in parallel pose (i.e., feet facing forward, approximately 8" apart) for 30

19 seconds [103] on a forceplate (AMTI MSA-6 MiniAmp strain gage amplifier) which captured

20 their center of pressure $(\mathrm{COP})$ path length $(\mathrm{cm})$ and velocity $(\mathrm{cm} / \mathrm{s})$. Signals from the forceplate

21 were collected at a sampling rate of $1000 \mathrm{~Hz}$. This was completed for four different trial types: 1)

22 eyes open standing directly on the forceplate (EOF; "firm surface"), 2) eyes open on a piece of

23 high-density foam placed on the forceplate (EOC; "compliant surface"; AIREX, Balance-Pad; 50 
$1 \times 41 \times 6 \mathrm{~cm}$; density $\left.=55 \mathrm{~kg} / \mathrm{m}^{2}\right), 3$ ) eyes closed on a compliant surface (ECC), 4) eyes closed on

2 a compliant surface while wearing noise-cancelling headphones for sound suppression (ECSS).

3 Participants wore a loose harness during the procedure to protect against falls.

$4 \quad$ Once collected, the first five seconds of the data were discarded [103]. The remaining

5 data were passed through a $2^{\text {nd }}$ order zero-lag dual-pass Butterworth filter with a $6 \mathrm{~Hz}$ cut-off

6 frequency. Mean COP path lengths, velocity, and velocity root-mean-square (RMS) [104]-[107]

7 were extracted from the data in MATLAB for each of the four trial types (recorded and analyzed

8 separately). COP path length was defined as the absolute length of sway in centimeters produced

9 by the participant during each of the conditions. Increased postural sway was therefore

10 associated with greater COP path lengths. Measures of velocity were obtained by taking the COP

11 excursion and dividing it by trial time, with poorer postural control being related to larger COP

12 velocity. To obtain velocity RMS, the square root of the mean of the squares of the velocity

13 measures were computed. Greater velocity RMS was related to more variable postural sway.

\section{Data Analysis}

16 All analyses were run using the threshold values obtained above in R 3.6.0 [108]. All data

17 were winsorized to treat potential outliers using the "DescTools" R package [109]. The data were

18 then evaluated for skewness using the "e1071" package [110] and evaluated for normality using

19 a generalized Shapiro-Wilk test for normality, then transformed to meet the Gaussian assumption

20 (although in-text means and standard deviation are calculated using raw, winsorized data to

21 facilitate comparisons in the literature). To compare the older and younger adults' vestibular

22 perceptual thresholds in each of the four vestibular perceptual tasks (heave and pitch detection

23 and discrimination) a series of four independent sample $t$-tests were conducted. Note that the 
1 deltas of the discrimination thresholds are reported here (e.g., instead of $22.6 \mathrm{~m} / \mathrm{s}^{2}$, we report the

2 delta: $2.6 \mathrm{~m} / \mathrm{s}^{2}$ ). We also calculate and report effect sizes (Cohen's $d$ ). Next, to compare COP

3 path length, velocity, and velocity-RMS across the four posturography tasks, between older and

4 younger adults we ran three separate 2 (Age Group: younger, older) $\times 4$ (Condition: EOF, EOC,

5 ECC, ECSS) mixed factorial ANOVA. Note that one younger adult participant's data was not

6 collected due to technical difficulties. Tukey-corrected post-hoc $t$-tests were used to explore

7 significant interaction effects. Greenhouse-Geisser corrections were applied to correct for

8 violations of sphericity.

9 We also conducted a series of Bonferroni-corrected Pearson correlations to assess the

10 extent to which COP path length (from each of the four posturography conditions) are associated

11 with each of the four vestibular perceptual thresholds. These correlations were conducted

12 separately for the older adult participant group and the younger adult participant group, to

13 compare whether these associations differed as a function of age.

\section{Results}

\section{Vestibular Psychophysics Task} greater heave detection thresholds $\left(M=0.022 \mathrm{~m} / \mathrm{s}^{2}, S D=0.051\right)$ than younger adults $(M=0.002$ $\left.\mathrm{m} / \mathrm{s}^{2}, S D=0.002, t(34.98)=-2.89, p=.007, d=0.554\right)($ Figure $3 \mathrm{~A})$. Further, older adults had

20 significantly greater pitch detection thresholds $\left(M=0.329 \mathrm{deg} / \mathrm{s}^{2}, S D=0.355, d=1.210\right)$ than

21 younger adults $\left(M=0.024 \mathrm{deg} / \mathrm{s}^{2}, S D=0.032, t(30.51)=-4.95, p<.001\right)$ (Figure 3B).

Discrimination Task. Independent sample $t$-tests showed that older adults $(M=0.241$

$\left.23 \mathrm{~m} / \mathrm{s}^{2}, S D=0.137\right)$ and younger adults $\left(M=0.211 \mathrm{~m} / \mathrm{s}^{2}, S D=0.119\right)$ did not have significantly 
1 different heave discrimination thresholds $(t(34.68)=-0.61, p=.549, d=0.234)$ (Figure 3C).

2 Likewise, there were no significant differences between pitch discrimination thresholds for older

$3\left(M=3.41 \mathrm{deg} / \mathrm{s}^{2}, S D=2.53\right)$ and younger adults $\left(M=2.89 \mathrm{deg} / \mathrm{s}^{2}, S D=2.25, t(31.23)=-0.783\right.$,

$4 p=.439, d=0.217)($ Figure 3D).
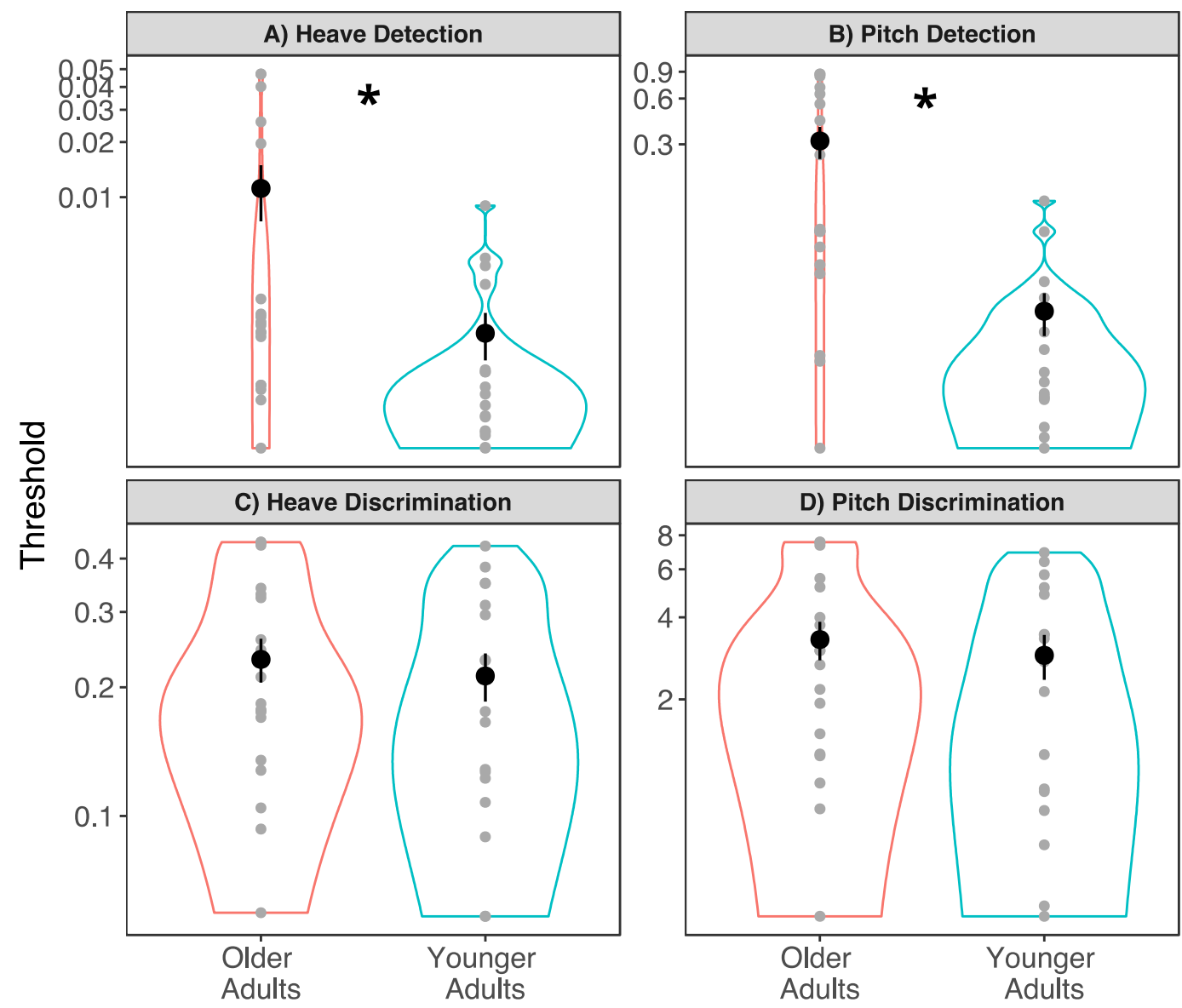

Figure 3. Heave and pitch detection and discrimination thresholds. $*=p<.05$. Graphs show data from the, A) Heave Detection condition, B) Pitch Detection condition, C) Heave

8 Discrimination condition, and d) Pitch Discrimination condition.

9 All data are plotted on logarithmic scales. Thresholds for heave data are in $\mathrm{m} / \mathrm{s}^{2}$, and for pitch 10 data in $\mathrm{deg} / \mathrm{s}^{2}$. Small points represent individual data. The larger black points represent the group 11 means and error bars are standard errors. The width of the borders of the violin plot represents 12 frequency count at each value on the y-axis. 


\section{Posturography}

A 2 (Age Group) $\times 4$ (Condition: EOF, EOC, ECC, ECSS) mixed factorial ANOVA, on

3 COP path length, revealed a significant main effect of Age Group, $F(1,34)=14.83, p<.001$,

4 indicating that older adults $(M=46.8 \mathrm{~cm}, S D=33.2)$ had significantly greater COP path lengths

5 than younger adults $(M=28.1 \mathrm{~cm}, S D=17.6)$ overall. The COP path lengths are shown for all

6 groups in Figure 4. There was also a significant main effect of Condition, $F(1.59,54.15)=$

7 105.24, $p<.001$, showing significant differences between all conditions $(p<.001)$, with the

8 exception of ECC relative to ECSS, $t(102)=-0.266, p=.993$. Specifically, easier posturography

9 conditions (e.g., EOC) showed smaller COP path lengths than more difficult posturography

10 conditions (e.g., ECC). There was also a significant Age Group $\times$ Condition interaction $(F(1.59$,

$1154.15)=5.50 p=.011$ ). Post-hoc $t$-tests showed that older adults had significantly greater COP

12 lengths relative to younger adults in the three hardest conditions: $\operatorname{EOC}(t(72,8)=-2.452, p$

$13=.017), \operatorname{ECC}(t(72.8)=-3.958, p<.001)$, and $\operatorname{ECSS}(t(72.8)=-4.818, p<.001)$, but not the EOF

14 condition $(p>.05)$.

15 These analyses were repeated with COP velocity $(\mathrm{m} / \mathrm{s})$, as well as COP velocity root-

16 mean-square (RMS). Similar results were observed (see Supplementary Materials, S.2, for full

17 statistical analyses), namely that COP velocity and velocity-RMS were larger in older adults

18 relative to younger adults, with similar differences between the conditions. Likewise, these

19 analyses revealed the same Group $\times$ Condition interaction, indicating greater velocity as well as

20 velocity-RMS in the three hardest conditions (EOC, ECC, and ECSS) for older adults relative to 21 younger adults. 


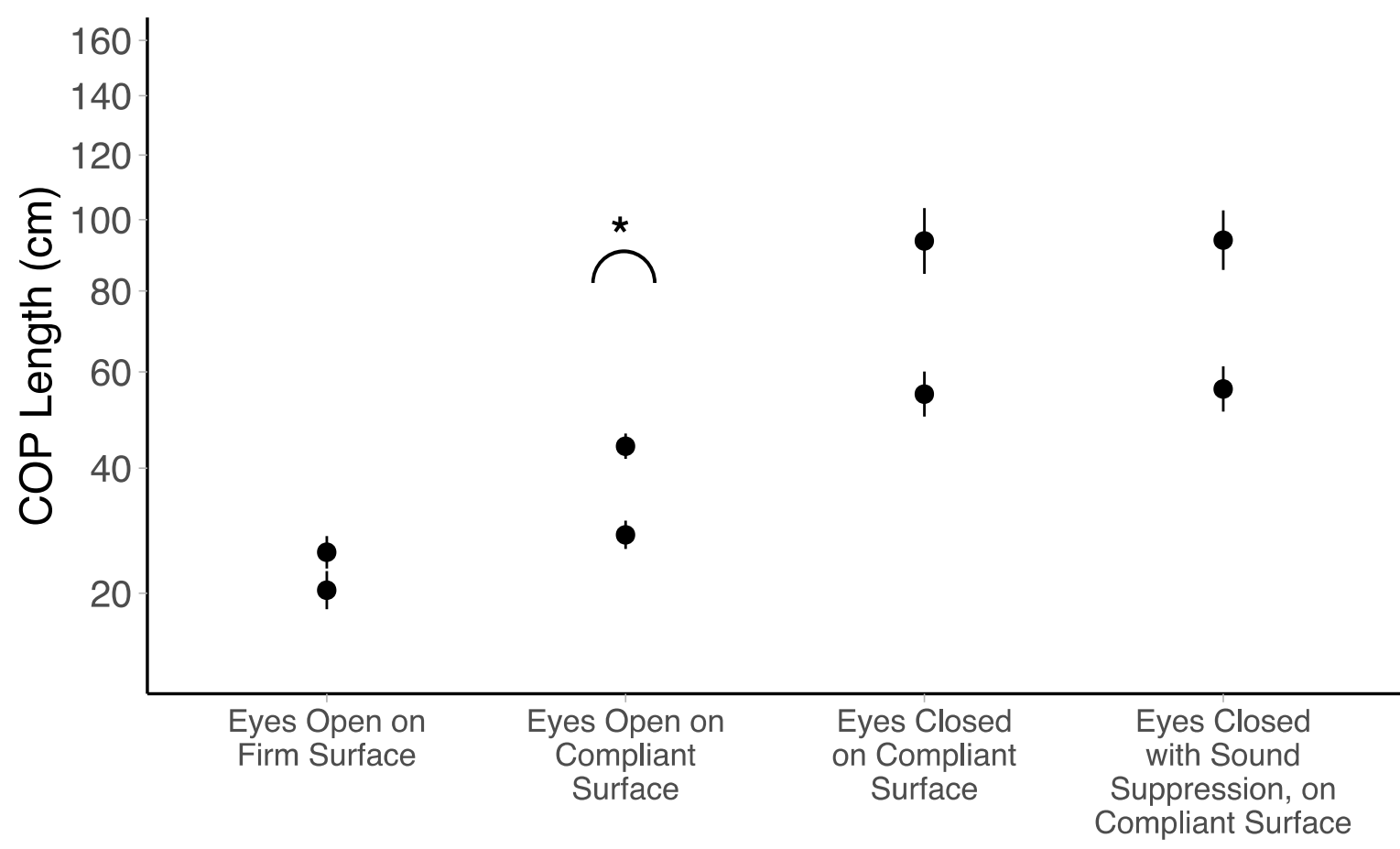

2 Figure 4. Posturography data for older and younger adults across all four conditions. ${ }^{*} p<.05$

3 Mean COP path length $(\mathrm{cm})$ are plotted (older adults $=$ solid red, younger adults $=$ dotted blue).

4 Individual participant data are plotted using single points (older adults $=$ red circles, younger

$5 \quad$ adults $=$ blue triangles). Data are plotted on a square-root scale.

7 Correlational Analyses between Vestibular Thresholds and Posturography

A series of Bonferroni-corrected Pearson correlations were used to examine the

9 relationship between the four postural task measures for COP path length and the four vestibular

10 perceptual threshold measures in older adults (Figure 5A) and younger adults (Figure 5B). Please

11 see the supplementary materials (S.3) for scatterplots, as well as Fisher's r-to-Z transformation to

12 compare the younger adult and older adults' correlations.

Perceptual Thresholds and Posturography. In older adults, heave detection thresholds

14 were positively correlated with COP path length for EOF $(r=.60, p<.007)$ and EOC $(r=.56, p$ 
$1=.013$ ) conditions, such that higher detection thresholds were associated with greater postural

2 sway (Figure 5A). Likewise, older adults' pitch detection thresholds were positively correlated

3 with COP path length for EOF $(r=.46, p<.048), \mathrm{EOC}(r=.59, p=.008), \mathrm{ECC}(r=.78, p$

$4<.001)$ and ECSS $(r=.82, p<.001)$ conditions. Finally, older adults' pitch discrimination

5 thresholds were positively associated with COP path length for the ECC $(r=.49, p<.033)$ and

6 ECSS conditions $(r=.64, p=.004)$, but there were no correlations between heave discrimination

7 and any measures of COP path length. For younger adults, there were no significant associations

8 observed between any of the posturography measures and vestibular perceptual thresholds

9 (Figure 5B).
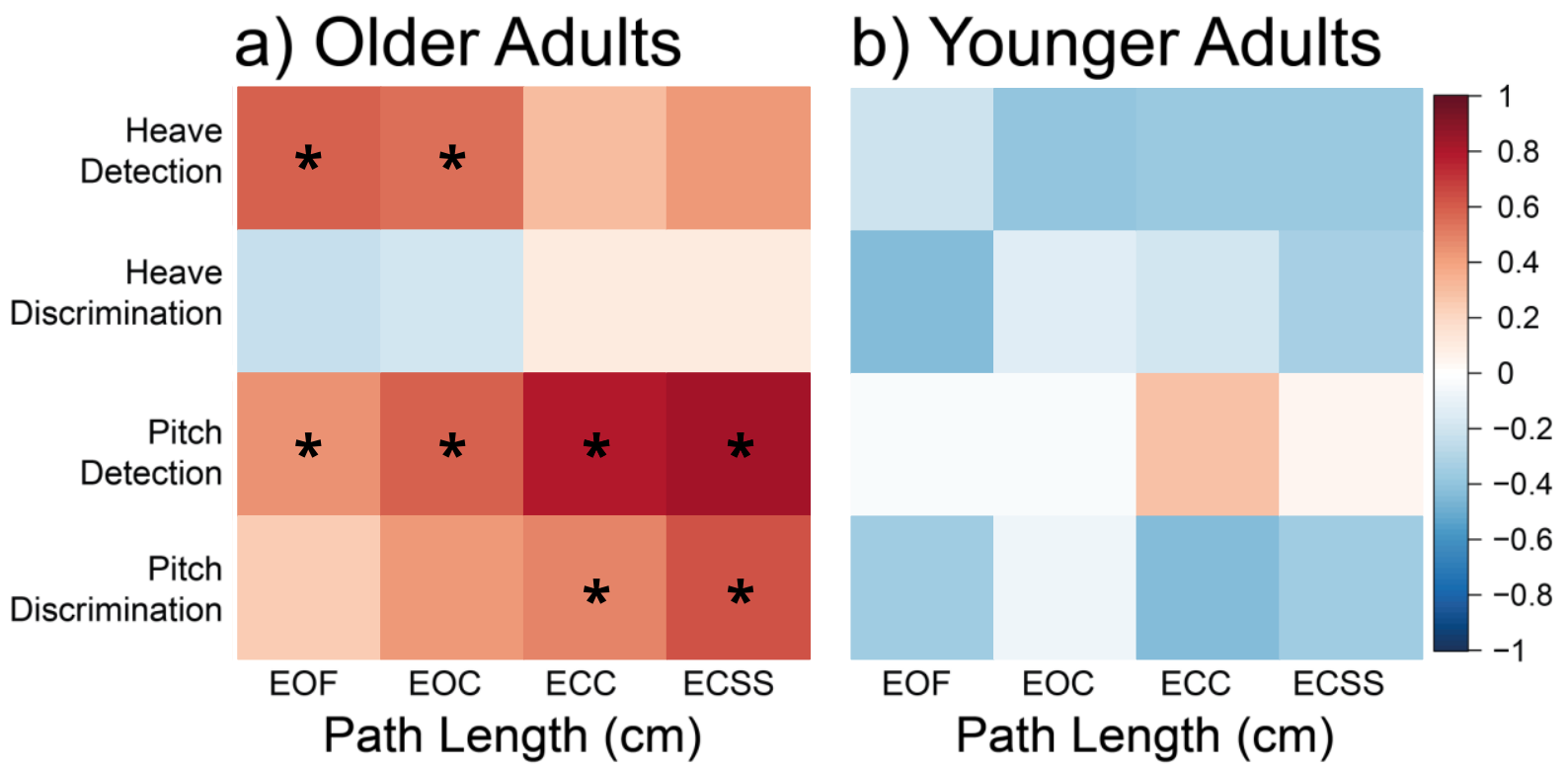

11 Figure 5. Correlation heatmaps. Graph illustrating the associations between posturography 12 measures (i.e., COP path length, $\mathrm{cm}$ ) and perceptual thresholds (i.e., $\mathrm{m} / \mathrm{s}^{2}$ for heave, and $\mathrm{deg} / \mathrm{s}^{2}$ 13 for pitch) in, A) older adults, and B) younger adults. Blue squares represent negative correlations 14 and red squares represent positive correlations. Lighter squares represent weaker correlations and darker squares represent stronger correlations. Correlations were Bonferroni-corrected. ${ }^{*} p<.05$.

Note. $\mathrm{EOF}=$ Eyes Open, Firm Surface; EOC = Eyes Open, Compliant Surface; ECC = Eyes 

11 different motion types and motion tasks. and pitch detection thresholds.

\section{Discussion}

We examined age-related changes to vestibular perceptual thresholds for heave and pitch motions. We also explored whether quiet standing balance performance was associated with vestibular perceptual thresholds in older or younger adults. We found that, compared to younger adults, older adults showed significantly higher vestibular detection thresholds in heave, and for the first time to our knowledge, in pitch. There were similar patterns of non-significant agerelated effects for the heave and pitch discrimination task thresholds, which may be a result of less robust age-related effects and/or insufficient power to observe these effects. Therefore, these results may be a conservative estimation of age-related changes in vestibular thresholds across

We also found that older adults had significantly greater COP path lengths relative to younger adults in all conditions except for the easiest condition (i.e., standing on a firm surface with eyes open). Importantly, we also found that, for older adults, but not younger adults, measures of postural sway (COP path length) were positively associated with vestibular perceptual thresholds, such that poorer balance (greater sway) was associated with higher heave

\section{Age-Related Changes in Pitch and Heave Perception}

Vestibular Detection Task. Older adults had higher heave and pitch detection thresholds than younger adults. These results are consistent with current studies in the literature that have also shown older age to be associated with increased direction discrimination (i.e., vestibular perceptual) thresholds in the heave direction [16], [21], [70], as well as in surge, sway, and rolltilt [16], [17], [19], [21], [70], but not yaw rotation [16], [70] (but see [18] who found no age 
1 differences in yaw motion perception during a two-interval detection and two-interval magnitude

2 discrimination task). Our study is the first to our knowledge to also examine age-related

3 differences in pitch perception in older and younger adults. Together these results suggest that

4 aging is associated with declines in a range of vestibular perceptual sensitivities across different

5 vestibular perception tasks and movement axes.

6 Histological, microscopic, and clinical assessments of vestibular functioning have long

7 associated the aging process with vestibular end-organ deterioration. For instance, aging has

8 been linked with significant declines in the number of hair cells, degeneration of the vestibular

9 ganglion, deterioration of the otoconia of the otoliths (see [6], [7], [27] for reviews), and reduced

10 oVEMP and cVEMP responses [8], [38], [96], [98]. There is also some evidence of age-related

11 decreases in vestibular cortical network connectivity [111], although other studies have shown

12 age-related central gains in vestibular perception, which seem to at least partially compensate for

13 peripheral declines in end-organ responsiveness [112].

14 Despite reporting no diagnosed vestibular impairments or chronic dizziness, some older

15 participants in this study showed evidence of end-organ dysfunction of the otoliths (VEMPs),

16 particularly the utricle (oVEMP), which is consistent with prior research [6], [8], [98], [113],

17 [114]. This may have contributed to older adults' reduced ability to detect pitch and heave

18 motions. It is important to note, however, that the recently described diagnostic criteria for

19 presbyvestibulopathy (PVP; age-related vestibular loss) includes measures of VOR gain (e.g.,

20 vHIT measures), but does not include VEMP responses [84]. It was concluded that, because an

21 absence of VEMP responses is frequently observed in older adults, the significance of these

22 absences for diagnostic purposes is not well understood. In our study, presence, or absence of a

23 VEMP response was not significantly correlated with heave or pitch detection thresholds in our 
1 participants (Table S.4.1, Supplementary Materials), except for cVEMP presence being

2 associated with reduced heave detection thresholds but only when not correcting for multiple

3 comparisons, $(r=-0.55, p=.042)$. Likewise, we did not observe a significant correlation

4 between VEMP presence and COP path length (Table S.4.2, Supplementary Materials).

5 Therefore, the extent to which VEMP responses might relate to observed age-related differences

6 in vestibular perceptual thresholds remains unclear.

7 Vestibular Discrimination Task. While we observed age-related differences in pitch and

8 heave detection thresholds, no age-related differences were observed for pitch or heave

9 discrimination tasks. This suggests that while older adults might have a preserved ability to

10 differentiate between two motions of similar magnitudes, their sensitivity to detecting these same

11 motions may be reduced relative to younger adults.

12 With regards to direction discrimination (which differs from this study's magnitude

13 discrimination task), previous research has found that older adults tend to demonstrate larger

14 thresholds than younger adults [16], [17], [19], [21], [70]. We suggest a number of possible

15 reasons for this discrepancy.

16 First, the nature of the task used in this study (magnitude discrimination) is different than

17 those used in the previous aging literature (direction-discrimination) where age-related

18 differences were found [16], [17], [19], [21], [70]. To our knowledge, only Chang and colleagues

19 investigated magnitude discrimination in older and younger adults, and also found no significant

20 differences [18]. Furthermore, these previous direction-discrimination tasks used a one-interval

21 forced choice task, whereas ours used a two-interval forced-choice task, which maybe have

22 further influenced threshold values. For instance, two-interval detection or discrimination tasks

23 such as those used in this current study, tend to lead to thresholds that are $1 / \sqrt{2}$ times smaller 
1 than 1-interval detection or discrimination tasks [102]. Such differences in magnitude may have

2 had an impact on the overall thresholds obtained by participants, leading to the null results

3 observed in this condition.

4 Differently, the frequency of the motion presented during vestibular perception tasks has

5 been found to influence whether age differences are observed. For instance, Roditi and Crane

6 [70] found that relative to younger adults, older adults had poorer surge direction discrimination

7 thresholds if motions were presented at $0.5 \mathrm{~Hz}$, but not if presented at $1 \mathrm{~Hz}$. Indeed, previous

8 studies have found that regardless of age, participants tend to show larger thresholds for higher

9 motion frequencies [100], [115], [116]. Once again, smaller thresholds obtained in both groups

10 may have eliminated previously observed age-differences.

11 Finally, the type of motion profile (e.g., single sine wave versus oscillating sinusoids),

12 may also affect threshold values and whether age-related differences are observed. Repeated

13 sine wave procedures offer a greater number of samples per trial to base one's perceptual

14 estimates, thereby leading to smaller thresholds (Figure 2). For example, Chang and colleagues

15 [18] and Bermùdez Rey and colleagues [16] evaluated magnitude discrimination (repeated sine

16 wave) and direction discrimination (single sine wave), respectively, in older adults, for yaw

17 rotation. Chang and colleagues' [18] older adults obtained thresholds which were almost half as

18 large $(0.81( \pm 0.42) \mathrm{deg} / \mathrm{sec})$ as those obtained by Bermùdez Rey and colleagues $(2016 ; 1.45$

19 (range: $1.14-1.84) \mathrm{deg} / \mathrm{s}$ ).

20 Ultimately, future studies could more systematically evaluate how different frequencies,

21 motion profiles, and tasks influence the extent to which age-related differences in vestibular

22 perception are observed. 
1 Associations between Vestibular Perceptual Thresholds and Postural Stability in Older and

2 Younger Adults

3 Our older adults were less stable than younger adults in all but the easiest (i.e., standing

4 on a firm surface with eyes open) balance conditions. Such age-related differences were

5 observed consistently, regardless of whether we examined COP path length, velocity, or velocity-

6 RMS. These results are consistent with previous literature showing that older adults demonstrate

7 greater COP path length, velocity, and variability compared to younger adults, especially when

8 multiple senses are impoverished or challenged (e.g., vision and proprioception; [10]-[12],

9 [117]. Postural control generally relies on the contributions and integration of visual, vestibular,

10 and somatosensory cues [117]-[120]. This process of multisensory integration during standing

11 balance is particularly relevant in the context of aging since older adults tend to show heightened

12 multisensory integration relative to younger adults [20], [121]-[125] and may weigh less reliable

13 sensory inputs more than is optimal [20], [126].

14 A novel finding of this study was that poorer vestibular perceptual sensitivity (i.e., higher

15 thresholds) was associated with greater postural sway, but only in older adults. These

16 associations were found particularly for pitch and heave detection (conditions which showed

17 significant age-related effects in the psychophysical tasks), as well as pitch discrimination.

18 Interestingly, for pitch detection and discrimination, the magnitude of the correlations increases

19 systematically as the difficulty of the postural task also increases. One possible reason for this

20 linear association is that the more difficult postural tasks also increased the need to rely on

21 vestibular input for balance maintenance (e.g., in the absence, or limited presence, of other

22 sensory cues). Specifically, standing on a compliant surface reduces the reliability of

23 somatosensory cues, closing the eyes eliminates the availability of visual cues, and auditory cues 
1 were reduced with sound suppression. As such, age-related declines in vestibular perceptual

2 sensitivity may be increasingly consequential to posture when other sensory inputs become more

3 impoverished. Importantly, we found fewer significant associations between postural stability

4 and vestibular discrimination thresholds. This lack of association may be due to the nature of the

5 posturography task (i.e., quiet standing). Specifically, given that precise discrimination estimates

6 may be especially important for responding to changes in balance/posture (as opposed to simply

7 maintaining stable balance/posture), using dynamic posturography tasks (e.g., recovery from

8 perturbation) in future may result in even stronger and more consistent associations.

9 With regards to the association between postural task difficulty and pitch detection and

10 discrimination, a possible explanation for this increased correlation with age might be related to

11 differences in vestibular end-organ functioning with age. For instance, semicircular canals -

12 necessary for pitch detection - show more age-related dysfunction in clinical tests than the

13 utricle or saccule [38], as well as a more pronounced loss of Type I hair cells [29], [32], [33], and

14 greater degeneration of the superior (relative to the inferior or posterior) vestibular nerve [36].

15 Such changes might reflect the extent to which pitch sensitivity contributes to postural stability

16 (e.g., COP path length) as one's reliance on vestibular information during standing balance

17 increases.

18 Importantly, no correlations between standing balance and vestibular perception were

19 observed in the younger adults. This is likely due to younger adult's more reliable vestibular

20 percepts and better ability to compensate for impoverished sensory conditions when maintaining

21 balance relative to older adults. 


\section{$1 \quad$ Limitations and Future Directions}

Participant Sample. Participants in this study underwent rigorous screening, which

3 allowed us to control for certain age-related factors, including for example, cognitive decline

4 [127]-[132], age-related hearing loss and tinnitus [1], [87], [89], [97], [133], diagnosed

5 vestibular disorders [134], and other serious health conditions. However, these common age-

6 related conditions are likely to also influence vestibular perception and postural control and, as

7 such, the sample in this study may not be representative of the typical older adult population.

8 Instead, these results may be a conservative estimate of age-related changes to vestibular

9 perception. Future studies can help disambiguate age-related changes in vestibular perceptual

10 sensitivity thresholds in a more representative sample of older adults by collecting data from

11 participants with a range of sensory, motor, and cognitive abilities. Furthermore, they can also

12 consider collecting additional baseline sensory, cognitive, and motor measures from both

13 samples of participants (older and younger) to determine whether there are associations among

14 these measures and experimentally test outcomes across the lifespan.

15 Extra-Vestibular Cues. It is also important to acknowledge the potential effect of extra-

16 vestibular cues on participants' detection and discrimination thresholds. Specifically, this study

17 was designed to reduce the influence of non-vestibular cues such as visual (blindfolds and

18 dimmed lights), auditory (white noise, passive noise-suppressing headphones), proprioceptive

19 (four-point harness, inflatable neck-pillow), and vibrotactile (padded seating and foam footrest) -

20 it remains possible that subtle vibration or proprioceptive cues may have still facilitated

21 perceptual judgements during the vestibular psychophysical tasks. . Access to these additional

22 non-vestibular sensory cues may account for the small perceptual thresholds we obtained,

23 particularly in the detection task - although some of these cues (e.g., vibration) could have also 
1 been detected by the vestibular system. Future studies could consider adding additional

2 vibrational masking noise during the tasks to further control for such additional cues, and better

3 account for this variability in our participants.

4 End-Organ Testing. In this study, we expected that older adults might demonstrate some 5 evidence of vestibular end-organ dysfunction [6]-[8], [38], [98], [113], [114] despite the absence

6 of a clinically diagnosed vestibular disorder [84]. To quantify such dysfunctions, we measured

7 canal and otolith functioning using VEMP and vHIT measures that included a particular set of

8 testing parameters (e.g., VEMP frequency of $500 \mathrm{~Hz}$ ), and further correlated these metrics to

9 measures of COP and vestibular thresholds. The lack of correlations of vestibular thresholds with

10 vHIT or VEMP suggests that common age-related declines in vestibular perceptual thresholds

11 are not due solely to end-organ or afferent deficits (see Agrawal et al., 2019 for a discussion on

12 the lack of association between measures of end-organ functioning and presbyvestibulopathy).

13 However, this choice of parameters may affect whether age-related differences are observed.

14 While for many older adults, VEMPs evoked at stimulus frequencies of $500 \mathrm{~Hz}$ show the greatest

15 response, many other older adults will only show evoked oVEMP or cVEMP responses at higher

16 tone burst frequencies (e.g., $750 \mathrm{~Hz}$ or $1000 \mathrm{~Hz}$ ). Future studies should more carefully evaluate

17 the associations between vHIT and VEMP measures and vestibular perceptual thresholds using a

18 range of end-organ testing parameters. Finally, future studies could also consider evaluating

19 VEMP and vHIT responses in the younger adult participant group to better understand changes

20 in the associations among end-organ functioning, vestibular perception, and postural control

21 across the lifespan. 
Conclusion

2 Older adults had higher heave and pitch detection thresholds than younger adults which,

3 together with pitch discrimination thresholds, were associated with increased postural sway (i.e.,

4 COP path length) particularly when sensory conditions were impoverished. These results could

5 have implications in the development of screening tools to detect mobility declines in older

6 adults, given that measurable declines in vestibular perceptual sensitivity were associated with

7 poorer postural stability. These convergent perceptual and behavioral measures may therefore

8 allow for better identification of falls risk.

9

10 


\section{References}

2 [1] G. A. Gabriel et al., "Vestibular Perceptual Thresholds in Older Adults With and Without Age-related Hearing Loss,” Ear Hear., vol. Publish Ah, 2021.

4 [2] Center for Disease Control, "10 Leading Causes of Injury Deaths by Age Group Highlighting Unintentional Injury Deaths, United States - 2018,” 2018.

6 [3] Center for Disease Control, "Leading Causes of Nonfatal Injury Reports,” 2018.

7 [4] K. Jahn, "The Aging Vestibular System: Dizziness and Imbalance in the Elderly," in Advances in Oto-Rhino-Laryngology, vol. 82, S. Karger AG, 2019, pp. 143-149.

9 [5] G. P. Jacobson, D. L. McCaslin, S. L. Grantham, and E. G. Piker, "Significant Vestibular System Impairment Is Common in a Cohort of Elderly Patients Referred for Assessment of Falls Risk,” J. Am. Acad. Audiol., vol. 19, no. 10, pp. 799-807, Nov. 2008.

6] D. Allen, L. Ribeiro, Q. Arshad, and B. M. Seemungal, “Age-related vestibular loss:

15 [7] E. Anson and J. J. Jeka, "Perspectives on aging vestibular function," Front. Neurol., vol. 6, 16 no. JAN, Jan. 2016.

17 [8] M. Maheu, M. S. Houde, S. P. Landry, and F. Champoux, "The effects of aging on clinical vestibular evaluations," Frontiers in Neurology, vol. 6, no. 205. Frontiers Media S.A., 2015.

F. B. Horak, "Postural orientation and equilibrium: What do we need to know about neural control of balance to prevent falls?," in Age and Ageing, 2006, vol. 35, no. SUPPL.2, p. 352.

23 [10] M. Hytönen, I. Pyykkö, H. Aalto, and J. Starck, "Postural control and age," Acta 
Otolaryngol., vol. 113, no. 1-2, pp. 119-122, 1993.

[11] I. Pyykko, P. Jantti, and H. Aalto, "Postural control in elderly subjects," Age Ageing, vol. 19, no. 3, pp. 215-221, May 1990.

[12] N. Teasdale, G. E. Stelmach, and A. Breunig, "Postural sway characteristics of the elderly under normal and altered visual and support surface conditions," Journals Gerontol., vol. 46, no. 6, pp. B238-B244, Nov. 1991.

[13] N. Teasdale, G. E. Stelmach, A. Breunig, and H. J. Meeuwsen, "Age differences in visual sensory integration,” Exp. Brain Res., vol. 85, no. 3, pp. 691-696, Jul. 1991.

[14] N. Teasdale and M. Simoneau, "Attentional demands for postural control: The effects of aging and sensory reintegration," Gait Posture, vol. 14, no. 3, pp. 203-210, Dec. 2001.

[15] S. L. Anacker, R. P. Di Fabio, and F. B. Horak, "Influence of sensory inputs on standing balance in community-dwelling elders with a recent history of falling," Phys. Ther., vol. 72, no. 8, pp. 575-584, Aug. 1992.

[16] M. C. Bermúdez Rey, T. K. Clark, W. Wang, T. Leeder, Y. Bian, and D. M. Merfeld, "Vestibular perceptual thresholds increase above the age of 40," Front. Neurol., vol. 7, no. OCT, p. 162, Oct. 2016.

[17] S. B. Beylergil, F. Karmali, W. Wang, M. C. Bermúdez Rey, and D. M. Merfeld, "Vestibular roll tilt thresholds partially mediate age-related effects on balance," in Progress in Brain Research, vol. 248, Elsevier B.V., 2019, pp. 249-267.

[18] N. Y. N. Chang et al., "Vestibular perception and the vestibulo-ocular reflex in young and older adults," Ear Hear., vol. 35, no. 5, pp. 565-570, 2014.

[19] F. Karmali, M. C. B. Rey, T. K. Clark, W. Wang, and D. M. Merfeld, "Multivariate analyses of balance test performance,vestibular thresholds, and age," Front. Neurol., vol. 
8, no. NOV, p. 578, Nov. 2017.

[20] R. Ramkhalawansingh, J. S. Butler, and J. L. Campos, "Visual-vestibular integration during self-motion perception in younger and older adults," Psychol. Aging, vol. 33, no. 5, pp. 798-813, Aug. 2018.

[21] M. J. Kobel, A. R. Wagner, D. M. Merfeld, and J. K. Mattingly, "Vestibular Thresholds: A Review of Advances and Challenges in Clinical Applications," Frontiers in Neurology, vol. 12. Frontiers, p. 643634, 19-Feb-2021.

[22] B. L. Day and R. C. Fitzpatrick, "The vestibular system.," Curr. Biol., vol. 15, no. 15, 2005.

[23] D. E. Angelaki and K. E. Cullen, "Vestibular system: The many facets of a multimodal sense," Annual Review of Neuroscience, vol. 31, no. 1. Annual Reviews, pp. 125-150, 17Jul-2008.

[24] U. Rosenhall, "Degenerative patterns in the aging human vestibular neuro-epithelia," Acta Otolaryngol., vol. 76, no. 1-6, pp. 208-220, 1973.

[25] U. Rosenhall and W. Rubin, "Degenerative changes in the human vestibular sensory epithelia," Acta Otolaryngol., vol. 79, no. 1-2, pp. 67-80, 1975.

[26] M. D. Ross, L. G. Johnsson, D. Peacor, and L. F. Allard, “Observations on normal and degenerating human otoconia," Ann. Otol. Rhinol. Laryngol., vol. 85, no. 3, pp. 310-326, May 1976.

[27] S. Iwasaki and T. Yamasoba, "Dizziness and imbalance in the elderly: Age-related decline in the vestibular system," Aging and Disease, vol. 6, no. 1. International Society on Aging and Disease, pp. 38-47, 2015.

[28] L. G. Johnsson and J. E. Hawkins, "Sensory and neural degeneration with aging, as seen 
in microdissections of the human inner ear," Ann. Otol. Rhinol. Laryngol., vol. 81, no. 2, pp. 179-193, 1972.

[29] S. D. Rauch, L. Velazquez-Villaseñor, P. S. Dimitri, and S. N. Merchant, "Decreasing Hair Cell Counts in Aging Humans," Ann. N. Y. Acad. Sci., vol. 942, no. 1, pp. 220-227, 2001.

[30] M. Anniko, “The aging vestibular hair cell,” Am. J. Otolaryngol. Neck Med. Surg., vol. 4, no. 3, pp. 151-160, 1983.

[31] M. Gleeson and H. Felix, "A comparative study of the effect of age on the human cochlear and vestibular neuroepithelia," Acta Otolaryngol., vol. 104, no. sup436, pp. 103-109, 1987.

[32] S. N. Merchant, L. Velázquez-Villaseñor, K. Tsuji, R. J. Glynn, C. Wall, and S. D. Rauch, "Temporal bone studies of the human peripheral vestibular system. Normative vestibular hair cell data.," Ann. Otol. Rhinol. Laryngol. Suppl., vol. 109, no. 5_suppl, pp. 3-13, 2000.

[33] A. J. Matheson, C. L. Darlington, and P. F. Smith, "Dizziness in the Elderly and Agerelated Degeneration of the Vestibular System," NZ. J. Psychol., 1999.

[34] L. Velázquez-Villaseñor, S. N. Merchant, K. Tsuji, R. J. Glynn, C. Wall, and S. D. Rauch, "Temporal bone studies of the human peripheral vestibular system. Normative Scarpa's ganglion cell data.," Ann. Otol. Rhinol. Laryngol. Suppl., 2000.

[35] J. J. Park, Y. Tang, I. Lopez, and A. Ishiyama, “Age-related change in the number of neurons in the human vestibular ganglion," J. Comp. Neurol., vol. 431, no. 4, pp. 437443, Mar. 2001.

[36] E. Richter, “Quantitative study of human scarpa's ganglion and vestibular sensory epithelia," Acta Otolaryngol., vol. 90, no. 1-6, pp. 199-208, 1980.

[37] K. D. Nguyen, M. S. Welgampola, and J. P. Carey, “Test-retest reliability and age-related 
characteristics of the ocular and cervical vestibular evoked myogenic potential tests.," Otol. Neurotol. Off. Publ. Am. Otol. Soc. Am. Neurotol. Soc. [and] Eur. Acad. Otol. Neurotol., vol. 31, no. 5, pp. 793-802, Jul. 2010.

[38] Y. Agrawal et al., "Decline in semicircular canal and otolith function with age," Otol. Neurotol., vol. 33, no. 5, pp. 832-839, 2012.

[39] A. J. Layman, C. Li, E. Simonsick, L. Ferrucci, J. P. Carey, and Y. Agrawal, “Association between saccular function and gait speed: Data from the Baltimore Longitudinal study of aging," Otol. Neurotol., vol. 32, no. 2, pp. 260-266, 2015.

[40] C. Li, A. J. Layman, J. P. Carey, and Y. Agrawal, "Epidemiology of vestibular evoked myogenic potentials: Data from the Baltimore Longitudinal Study of Aging," Clin. Neurophysiol., vol. 126, no. 11, pp. 2207-2215, Nov. 2015.

[41] E. Matiño-Soler, E. Esteller-More, J. C. Martin-Sanchez, J. M. Martinez-Sanchez, and N. Perez-Fernandez, "Normative data on angular vestibulo-ocular responses in the yaw axis measured using the video head impulse test," Otol. Neurotol., vol. 36, no. 3, pp. 466-471, 2015.

[42] L. A. McGarvie, H. G. MacDougall, G. M. Halmagyi, A. M. Burgess, K. P. Weber, and I. S. Curthoys, "The video head impulse test (vHIT) of semicircular canal function - agedependent normative values of VOR gain in healthy subjects," Front. Neurol., vol. 6, no. JUL, p. 1, Jul. 2015.

[43] W. V. C. Figtree, J. C. Menant, A. T. Chau, P. P. Hübner, S. R. Lord, and A. A. Migliaccio, "Prevalence of Vestibular Disorders in Independent People Over 50 That Experience Dizziness," Front. Neurol., vol. 0, p. 709, May 2021.

[44] E. Anson, R. T. Bigelow, S. Studenski, N. Deshpande, and Y. Agrawal, "Failure on the 
Foam Eyes Closed Test of Standing Balance Associated With Reduced Semicircular Canal Function in Healthy Older Adults," Ear Hear., vol. 40, no. 2, pp. 340-344, Mar. 2019.

[45] E. Anson et al., "Loss of peripheral sensory function explains much of the increase in postural sway in healthy older adults," Front. Aging Neurosci., vol. 9, no. JUN, p. 202, Jun. 2017.

[46] Y. Agrawal, M. Davalos-Bichara, M. G. Zuniga, and J. P. Carey, "Head impulse test abnormalities and influence on gait speed and falls in older individuals," Otol. Neurotol., vol. 34, no. 9, pp. 1729-1735, 2013.

[47] M. Y. Osoba, A. K. Rao, S. K. Agrawal, and A. K. Lalwani, "Balance and gait in the elderly: A contemporary review," Laryngoscope Investig. Otolaryngol., vol. 4, no. 1, pp. 143-153, Feb. 2019.

[48] B. E. Maki and W. E. McIlroy, "Postural control in the older adult," Clinics in Geriatric Medicine, vol. 12, no. 4. W.B. Saunders, pp. 635-658, 01-Nov-1996.

[49] J. R. Mahoney, K. Cotton, J. Verghese, and A. Newman, "Multisensory Integration Predicts Balance and Falls in Older Adults," Journals Gerontol. - Ser. A Biol. Sci. Med. Sci., vol. 74, no. 9, pp. 1429-1435, Aug. 2019.

[50] J. R. Mahoney and J. Verghese, "Does cognitive impairment influence visualsomatosensory integration and mobility in older adults?," Journals Gerontol. - Ser. A Biol. Sci. Med. Sci., vol. 75, no. 3, pp. 581-588, Feb. 2020.

[51] E. A. Maylor and A. M. Wing, "Age differences in postural stability are increased by additional cognitive demands," Journals Gerontol. - Ser. B Psychol. Sci. Soc. Sci., vol. 51, no. 3, pp. 143-154, May 1996.

[52] M. Hytönen, I. Pyykkö, H. Aalto, and J. Starck, "Postural control and age," Acta 
Otolaryngol., vol. 113, no. 1-2, pp. 119-122, 1993.

[53] M. H. Woollacott, A. Shumway-Cook, and L. M. Nashner, "Aging and posture control: Changes in sensory organization and muscular coordination," Int. J. Aging Hum. Dev., vol. 23, no. 2, pp. 97-114, Sep. 1986.

[54] D. Manchester, M. Woollacott, N. Zederbauer-Hylton, and O. Marin, "Visual, vestibular and somatosensory contributions to balance control in the older adult," Journals Gerontol., vol. 44, no. 4, pp. M118-M127, Jul. 1989.

[55] L. Grabherr, K. Nicoucar, F. W. Mast, and D. M. Merfeld, "Vestibular thresholds for yaw rotation about an earth-vertical axis as a function of frequency," Exp. Brain Res., vol. 186, no. 4, pp. 677-681, Apr. 2008.

[56] J. S. Butler, J. L. Campos, and H. H. Bülthoff, "Optimal visual-vestibular integration under conditions of conflicting intersensory motion profiles," Exp. Brain Res., vol. 233, no. 2, pp. 587-597, 2015.

[57] J. S. Butler, J. L. Campos, H. H. Bülthoff, and S. T. Smith, "The role of stereo vision in visual-vestibular integration," Seeing Perceiving, vol. 24, no. 5, pp. 453-470, 2011.

[58] J. S. Butler, S. T. Smith, J. L. Campos, and H. H. Bülthoff, "Bayesian integration of visual and vestibular signals for heading," J. Vis., vol. 10, no. 11, pp. 23-23, Sep. 2010.

[59] J. L. Campos, J. S. Butler, and H. H. Bülthoff, "Multisensory integration in the estimation of walked distances," Exp. Brain Res., vol. 218, no. 4, pp. 551-565, May 2012.

[60] B. T. Crane, "Direction Specific Biases in Human Visual and Vestibular Heading Perception," PLoS One, vol. 7, no. 12, p. e51383, Dec. 2012.

[61] L. R. Harris, M. Jenkin, and D. C. Zikovitz, "Visual and non-visual cues in the perception of linear self motion," Exp. Brain Res., vol. 135, no. 1, pp. 12-21, 2000. 
1 [62] P. M. Jaekl, M. R. Jenkin, and L. R. Harris, "Perceiving a stable world during active rotational and translational head movements," Exp. Brain Res., vol. 163, no. 3, pp. 388$399,2005$.

[63] C. R. Fetsch, A. H. Turner, G. C. DeAngelis, and D. E. Angelaki, "Dynamic Reweighting of Visual and Vestibular Cues during Self-Motion Perception," J. Neurosci., vol. 29, no. 49, pp. 15601-15612, 2009.

[64] I. Frissen, J. L. Campos, J. L. Souman, and M. O. Ernst, "Integration of vestibular and proprioceptive signals for spatial updating,” Exp. Brain Res., vol. 212, no. 2, pp. 163-176, Jul. 2011.

[65] J. L. Campos, J. H. Siegle, B. J. Mohler, H. H. Bülthoff, and J. M. Loomis, "Imagined Self-Motion Differs from Perceived Self-Motion: Evidence from a Novel Continuous Pointing Method," PLoS One, vol. 4, no. 11, p. e7793, Nov. 2009.

[66] J. H. Siegle, J. L. Campos, B. J. Mohler, J. M. Loomis, and H. H. Bülthoff, "Measurement of instantaneous perceived self-motion using continuous pointing," Exp. Brain Res., vol. 195, no. 3, pp. 429-444, May 2009.

[67] C. Pfeiffer, A. Serino, and O. Blanke, "The vestibular system: A spatial reference for bodily self-consciousness," Frontiers in Integrative Neuroscience, vol. 8, no. APR. Frontiers Research Foundation, p. 31, 17-Apr-2014.

[68] B. L. Matthews, J. H. Ryu, and C. Bockaneck, "Vestibular contribution to spatial orientation," Acta Otolaryngol., vol. 108, no. S468, pp. 149-154, 1989.

[69] D. E. Angelaki, E. M. Klier, and L. H. Snyder, “A Vestibular Sensation: Probabilistic Approaches to Spatial Perception," Neuron, vol. 64, no. 4. Cell Press, pp. 448-461, 25Nov-2009. 
1 [70] R. E. Roditi and B. T. Crane, "Directional asymmetries and age effects in human selfmotion perception," JARO - J. Assoc. Res. Otolaryngol., vol. 13, no. 3, pp. 381-401, Jun. 2012.

[71] B. M. Seemungal, I. A. Gunaratne, I. O. Fleming, M. A. Gresty, and A. M. Bronstein, "Perceptual and nystagmic thresholds of vestibular function in yaw," J. Vestib. Res., vol. 14, no. 6, pp. 461-466, Jan. 2004.

[72] G. Barbieri, A. S. Gissot, and D. Pérennou, "Ageing of the postural vertical," Age (Omaha)., vol. 32, no. 1, pp. 51-60, 2010.

[73] P. Manckoundia, F. Mourey, D. Pérennou, and P. Pfitzenmeyer, "Backward disequilibrium in elderly subjects," Clin. Interv. Aging, vol. 3, no. 4, p. 667, 2008.

[74] P. Manckoundia, F. Mourey, P. Pfitzenmeyer, J. Van Hoecke, and D. Pérennou, “Is backward disequilibrium in the elderly caused by an abnormal perception of verticality? A pilot study," Clin. Neurophysiol., vol. 118, no. 4, pp. 786-793, Apr. 2007.

[75] A. J. Van den Bogert, M. J. Pavol, and M. D. Grabiner, "Response time is more important than walking speed for the ability of older adults to avoid a fall after a trip," J. Biomech., vol. 35, no. 2, pp. 199-205, 2002.

[76] P. E. Roos and J. B. Dingwell, "Using dynamic walking models to identify factors that contribute to increased risk of falling in older adults," Hum. Mov. Sci., vol. 32, no. 5, pp. 984-996, Oct. 2013.

[77] T. De Boer, M. Wisse, and F. C. T. Van Der Helm, "Mechanical analysis of the preferred strategy selection in human stumble recovery," J. Biomech. Eng., vol. 132, no. 7, Jul. 2010.

[78] P. E. Roos, M. P. McGuigan, and G. Trewartha, "The role of strategy selection, limb force 
capacity and limb positioning in successful trip recovery," Clin. Biomech., vol. 25, no. 9, pp. 873-878, Nov. 2010.

[79] F. Karmali, A. D. Goodworth, Y. Valko, T. Leeder, R. J. Peterka, and D. M. Merfeld, “The role of vestibular cues in postural sway," J. Neurophysiol., vol. 125, no. 2, pp. 672-686, 2021.

[80] L. Prosperini and C. Pozzilli, "The Clinical Relevance of Force Platform Measures in Multiple Sclerosis: A Review,” Mult. Scler. Int., vol. 2013, pp. 1-9, 2013.

[81] R. W. Baloh, K. M. Jacobson, K. Beykirch, and V. Honrubia, "Static and dynamic posturography in patients with vestibular and cerebellar lesions," Arch. Neurol., vol. 55, no. 5, pp. 649-654, May 1998.

[82] N. E. Wolter et al., "BalanCI: Head-referenced cochlear implant stimulation improves balance in children with bilateral cochleovestibular loss," Audiol. Neurotol., vol. 25, no. 1-2, pp. 60-71, Feb. 2020.

[83] N. E. Wolter et al., "Unilateral hearing loss is associated with impaired balance in children: A pilot study,” Otol. Neurotol., vol. 37, no. 10, pp. 1589-1595, Nov. 2016.

[84] Y. Agrawal et al., "Presbyvestibulopathy: Diagnostic criteria Consensus document of the classification committee of the Bárány Society," J. Vestib. Res. Equilib. Orientat., vol. 29, no. 4, pp. 161-170, Jan. 2019.

[85] Z. S. Nasreddine et al., "The Montreal Cognitive Assessment, MoCA: A brief screening tool for mild cognitive impairment," J. Am. Geriatr. Soc., vol. 53, no. 4, pp. 695-699, Apr. 2005.

[86] J. L. Campos, R. Ramkhalawansingh, and M. K. Pichora-Fuller, "Hearing, self-motion perception, mobility, and aging," Hear. Res., vol. 369, pp. 42-55, Mar. 2018. 
1 [87] M. G. Carpenter and J. L. Campos, "The effects of hearing loss on balance: a critical review.," Ear Hear., vol. 41, pp. 107S-119S, 2020.

[88] F. R. Lin and L. Ferrucci, "Hearing loss and falls among older adults in the United States," Arch. Intern. Med., vol. 172, no. 4, pp. 369-371, Feb. 2012.

[89] A. V. Lubetzky, M. Gospodarek, L. Arie, J. Kelly, A. Roginska, and M. Cosetti, “Auditory Input and Postural Control in Adults: A Narrative Review," JAMA Otolaryngology - Head and Neck Surgery. American Medical Association, pp. E1-E8, 2020.

[90] A. Viljanen et al., "Hearing as a predictor of falls and postural balance in older female twins," Journals Gerontol. - Ser. A Biol. Sci. Med. Sci., vol. 64, no. 2, pp. 312-317, 2009.

[91] M. G. Zuniga et al., "Association between Hearing Loss and Saccular Dysfunction in Older Individuals,” Otol. Neurotol., vol. 33, no. 9, pp. 1586-1592, Dec. 2012.

[92] ISO 8253-1., “Acoustics-Audiometric test methods-Part 1: Basic pure tone air and bone conduction threshold audiometry." Geneva: International Organization for Standardization., 1989.

[93] H. G. MacDougall, L. A. McGarvie, G. M. Halmagyi, I. S. Curthoys, and K. P. Weber, “Application of the Video Head Impulse Test to Detect Vertical Semicircular Canal Dysfunction,” Otol. Neurotol., vol. 34, no. 6, pp. 974-979, Aug. 2013.

[94] G. M. Halmagyi, L. Chen, H. G. MacDougall, K. P. Weber, L. A. McGarvie, and I. S. Curthoys, “The video head impulse test," Front. Neurol., vol. 8, no. JUN, p. 1, Jun. 2017.

[95] K. L. Janky, J. N. Patterson, N. T. Shepard, M. L. A. Thomas, and J. A. Honaker, "Effects of device on video head impulse test (vHIT) gain," Journal of the American Academy of Audiology, vol. 28, no. 9. American Academy of Audiology, pp. 778-785, 01-Oct-2017.

[96] K. Brantberg, "Vestibular evoked myogenic potentials (VEMPs): Usefulness in clinical 
neurotology," Seminars in Neurology, vol. 29, no. 5. (C Thieme Medical Publishers, pp. 541-547, 15-Nov-2009.

[97] C. Li, M. G. Zuniga, K. D. Nguyen, J. P. Carey, and Y. Agrawal, "How to interpret latencies of cervical and ocular vestibular-evoked myogenic potentials: Our experience in fifty-three participants," Clinical Otolaryngology, vol. 39, no. 5. Blackwell Publishing Ltd, pp. 297-301, 2014.

[98] E. G. Piker, G. P. Jacobson, R. F. Burkard, D. L. McCaslin, and L. J. Hood, "Effects of age on the tuning of the cVEMP and oVEMP," Ear Hear., vol. 34, no. 6, Nov. 2013.

[99] L. E. Powell and A. M. Myers, "The Activities-Specific Balance Confidence (ABC) scale," Journals Gerontol. - Ser. A Biol. Sci. Med. Sci., vol. 50A, no. 1, pp. M28-M34, Jan. 1995.

[100] A. R. Naseri and P. R. Grant, "Human discrimination of translational accelerations," Exp. Brain Res., vol. 218, no. 3, pp. 455-464, May 2012.

[101] M. M. Taylor and C. D. Creelman, "PEST: Efficient Estimates on Probability Functions," J. Acoust. Soc. Am., vol. 41, no. 4A, pp. 782-787, Apr. 1967.

[102] D. M. Merfeld, "Signal detection theory and vestibular thresholds: I. Basic theory and practical considerations," in Experimental Brain Research, 2011, vol. 210, no. 3-4, pp. 389-405.

[103] F. Scoppa, R. Capra, M. Gallamini, and R. Shiffer, "Clinical stabilometry standardization. Basic definitions - Acquisition interval - Sampling frequency.," Gait Posture, vol. 37, no. 2, pp. 290-292, Feb. 2013.

[104] T. Paillard and F. Noé, “Techniques and Methods for Testing the Postural Function in Healthy and Pathological Subjects," BioMed Research International, vol. 2015. Hindawi 
Publishing Corporation, 2015.

[105] T. E. Prieto, J. B. Myklebust, R. G. Hoffmann, E. G. Lovett, and B. M. Myklebust, "Measures of postural steadiness: Differences between healthy young and elderly adults," IEEE Trans. Biomed. Eng., vol. 43, no. 9, pp. 956-966, Sep. 1996.

[106] Z. Li, Y. Y. Liang, L. Wang, J. Sheng, and S. J. Ma, "Reliability and validity of center of pressure measures for balance assessment in older adults," J. Phys. Ther. Sci., vol. 28, no. 4, pp. 1364-1367, Apr. 2016.

[107] M. Moghadam et al., "Reliability of center of pressure measures of postural stability in healthy older adults: Effects of postural task difficulty and cognitive load," Gait Posture, vol. 33, no. 4, pp. 651-655, Apr. 2011.

[108] R Core Team, “A language and environment for statistical computing.” R Foundation for Statistical Computing; 2017, Vienna, Austria, 2017.

[109] A. Signorell et al., "DescTools: Tools for descriptive statistics.” 2019.

[110] Meyer, Dimitriadou, Hornik, Weingessel, and Leisch, “e1071: Misc Functions of the Department of Statistics.” Probability Theory Group (Formerly: E1071), TU Wien, 2018.

[111] C. A. M. Cyran, R. Boegle, T. Stephan, M. Dieterich, and S. Glasauer, “Age-related decline in functional connectivity of the vestibular cortical network," Brain Struct. Funct., vol. 221, no. 3, pp. 1443-1463, Apr. 2016.

[112] R. M. Peters, J. S. Blouin, B. H. Dalton, and J. T. Inglis, “Older adults demonstrate superior vestibular perception for virtual rotations," Exp. Gerontol., vol. 82, pp. 50-57, Sep. 2016.

[113] H. C. Su, T. W. Huang, Y. H. Young, and P. W. Cheng, “Aging effect on vestibular evoked myogenic potential," Otol. Neurotol., vol. 25, no. 6, pp. 977-980, Nov. 2004. 
1 [114] C. L. Tseng, C. H. Chou, and Y. H. Young, "Aging effect on the ocular vestibular-evoked myogenic potentials," Otol. Neurotol., vol. 31, no. 6, pp. 959-963, Aug. 2010.

[115] A. J. Benson, M. B. Spencer, and J. R. Stott, "Thresholds for the detection of the direction of whole-body, linear movement in the horizontal plane," Aviat. Space. Environ. Med., vol. 57, no. 11, pp. 1088-1096, 1986.

[116] A. J. Gundry, "Thresholds of perception for periodic linear motion.," Aviat. Space. Environ. Med., vol. 49, no. 5, pp. 679-686, 1978.

[117] R. J. Peterka, "Sensorimotor integration in human postural control," J. Neurophysiol., vol. 88, no. 3, pp. 1097-1118, 2002.

[118] A. M. Bronstein, "Multisensory integration in balance control," in Handbook of Clinical Neurology, vol. 137, Elsevier B.V., 2016, pp. 57-66.

[119] L. Assländer and R. J. Peterka, "Sensory reweighting dynamics in human postural control," J. Neurophysiol., vol. 111, no. 9, pp. 1852-1864, May 2014.

[120] Y. Ivanenko and V. S. Gurfinkel, "Human postural control," Frontiers in Neuroscience, vol. 12, no. MAR. Frontiers Media S.A., p. 171, 20-Mar-2018.

[121] A. L. de Dieuleveult, P. C. Siemonsma, J. B. F. van Erp, and A.-M. Brouwer, "Effects of Aging in Multisensory Integration: A Systematic Review," Front. Aging Neurosci., vol. 9, no. 80, Mar. 2017.

[122] J. L. Mozolic, C. E. Hugenschmidt, A. M. Peiffer, and P. J. Laurienti, "Multisensory integration and aging," in The Neural Bases of Multisensory Processes, CRC Press, 2011, pp. 381-392.

[123] D. J. DeLoss, R. S. Pierce, and G. J. Andersen, "Multisensory integration, aging, and the sound-induced flash illusion," Psychol. Aging, vol. 28, no. 3, pp. 802-812, Sep. 2013. 
1 [124] P. J. Laurienti, J. H. Burdette, J. A. Maldjian, and M. T. Wallace, "Enhanced multisensory integration in older adults," Neurobiol. Aging, vol. 27, no. 8, pp. 1155-1163, Aug. 2006.

[125] J. Lupo and M. Barnett-Cowan, "Impaired perceived timing of falls in the elderly," Gait Posture, vol. 59, pp. 40-45, Jan. 2018.

[126] J. R. Berard, J. Fung, and A. Lamontagne, "Impact of aging on visual reweighting during locomotion," Clin. Neurophysiol., vol. 123, no. 7, pp. 1422-1428, Jul. 2012.

[127] S. Carr, M. K. Pichora-Fuller, K. Z. H. Li, N. Phillips, and J. L. Campos, "Multisensory, multi-Tasking performance of older adults with and without subjective cognitive decline," Multisens. Res., vol. 32, no. 8, pp. 797-829, Dec. 2019.

[128] S. Carr, K. Z. Li, M. K. Pichora-Fuller, and J. L. Campos, "Effects of age on listening and postural control during realistic multitasking conditions.," Hum. Mov. Sci., vol. 73, p. 102664, 2020.

[129] V. Nieborowska, S. T. Lau, J. L. Campos, M. K. Pichora-Fuller, A. Novak, and K. Z. H. Li, "Effects of Age on Dual-Task Walking While Listening," J. Mot. Behav., vol. 51, no. 4, pp. 416-427, Jul. 2019.

[130] K. C. Moylan and E. F. Binder, "Falls in older adults: risk assessment, management and prevention.," Am. J. Med., vol. 120, no. 6, pp. 493.e1-e6, 2007.

[131] M. Leandri et al., "Balance features in Alzheimer's disease and amnestic mild cognitive impairment," J. Alzheimer's Dis., vol. 16, no. 1, pp. 113-120, Jan. 2009.

[132] K. Z. H. Li and U. Lindenberger, "Relations between aging sensory/sensorimotor and cognitive functions," Neurosci. Biobehav. Rev., vol. 26, no. 7, pp. 777-783, Nov. 2002.

[133] G. M. S. Abdel-Salam, "The association between age-related sensorineural hearing loss and saccular dysfunction in the elderly," Saudi J. Otorhinolaryngol. Head Neck Surg., vol. 


1

22, no. 1, pp. 7-12, 2020.

[134] Y. Agrawal, P. F. Smith, and P. B. Rosenberg, "Vestibular impairment, cognitive decline and Alzheimer's disease: balancing the evidence," Aging and Mental Health, vol. 24, no. 5. Routledge, pp. 705-708, 03-May-2020.

\section{Author Contributions Statement}

G.A.G., J.J.G., and J.L.C. conceived the study. L.R.H., J.J.G., S.L.C., K.A.G., B.C.H., and J.L.C. helped designed the study. G.A.G. and J.J.G. collected the data. G.A.G., L.R.H., and J.L.C., analyzed and interpreted the data. G.A.G. and J.L.C. drafted the article, and all authors contributed to critically revising the article.

(1)

\section{Additional Information}

J.L.C. has received a NSERC Discovery Grant, and G.A.G. has received a NSERC PGS-D. The authors have no conflict of interest to declare.

\section{Legends}

Table 1. Summary of baseline assessments measured in the older adult participants

${ }^{1} \mathrm{PTA}=$ Pure Tone Average; frequencies tested: 500, 1000, 2000, and $4000 \mathrm{~Hz}$, inclusive, with a cut-off threshold above $25 \mathrm{~dB}$ HL.

${ }^{2} \mathrm{MoCA}=$ Montreal Cognitive Assessment (max score $=30$; clinical cut off $\leq 26$ pts)

${ }^{3} \mathrm{vHIT}=$ Video Head Impulse Test. 9 participants were not able to come back to complete this session. Median gain for $60 \mathrm{~ms}$ reported. One participant obtained a median gain below the 0.7 cut off score at $60 \mathrm{~ms}$ (they obtained 0.67 , in the left ear).

${ }^{4} \mathrm{cVEMP}=5$ participants were not able to come back to complete this session. Cervical Vestibular Evoked Myogenic Potential

${ }^{5} \mathrm{OVEMP}=5$ participants were not able to come back to complete this session. Ocular Vestibular Evoked Myogenic Potential

${ }^{6} \mathrm{ABC}=$ Activities-specific Balance Confidence Scale $(\max$ score $=100 \%)$ 
Figure 3. Vestibular Psychophysics setup.

Schematic of the laboratory setup for the psychophysical task including the 6-degrees-offreedom motion platform.

\section{Table 2. Initial peak accelerations used for the psychophysical tasks.}

Note. The initial comparison movements in this table represent the initial acceleration values.

These values changed throughout the session as a function of the PEST procedure outlined in the text.

Figure 4. Diagrammatic representation of a single heave discrimination trial. A) the position (m) relative to the upright start position, B) the velocity $(\mathrm{m} / \mathrm{s})$ and C) the acceleration $\left(\mathrm{m} / \mathrm{s}^{2}\right)$ of the motion base. For pitch trials, displacement was measured in degrees, velocity in $\mathrm{deg} / \mathrm{s}$, and acceleration in $\mathrm{deg} / \mathrm{s}^{2}$. The yellow shaded area highlights the first movement $(5 \mathrm{~s}$; here, standard movement), the grey shaded area represents the fade-in between the first and second movement (3s), and the blue region represents the second movement ( $5 \mathrm{~s}$; here, comparison movement). The unshaded white regions represent the fade from no motion to the first movement (3s), or from the second movement to no motion (3s), and rest (1s).

Figure 3. Heave and pitch detection and discrimination thresholds. $*=p<.05$. Graphs show data from the, A) Heave Detection condition, B) Pitch Detection condition, C) Heave Discrimination condition, and d) Pitch Discrimination condition. Data are plotted on logarithmic scales. Thresholds for heave data are in $\mathrm{m} / \mathrm{s} 2$, and for pitch data in $\mathrm{deg} / \mathrm{s} 2$. Small points represent individual data. The larger black points represent the group means and error bars are standard errors. The width of the borders of the violin plot represents frequency count at each value on the y-axis.

Figure 4. Posturography data for older and younger adults across all four conditions. ${ }^{*} p<.05$ Mean COP path length $(\mathrm{cm})$ are plotted (older adults $=$ solid red, younger adults $=$ dotted blue). Individual participant data are plotted using single points (older adults $=$ red circles, younger adults $=$ blue triangles). Data are plotted on a square-root scale.

Figure 5. Correlation heatmaps. Graph illustrating the associations between posturography measures (i.e., COP path length, $\mathrm{cm}$ ) and perceptual thresholds (i.e., $\mathrm{m} / \mathrm{s}^{2}$ for heave, and $\mathrm{deg} / \mathrm{s}^{2}$ for pitch) in, A) older adults, and B) younger adults. Blue squares represent negative correlations and red squares represent positive correlations. Lighter squares represent weaker correlations and darker squares represent stronger correlations. Correlations were Bonferroni-corrected. ${ }^{*} p<.05$. 


\section{Supplementary Files}

This is a list of supplementary files associated with this preprint. Click to download.

- GabrielSupplementalMaterialsSciReports.docx 